\title{
Equal-Time Commutators and Electromagnetic Mass Splittings
}

\author{
David G. Boulware* $\dagger$ \\ International Centre for Theoretical Physics, Trieste, Italy \\ AND \\ S. DESER $\ddagger$ \\ Physics Department, Brandeis University, Waltham, Massachusetts 02154
}

(Received 17 June 1968)

\begin{abstract}
The relation between electromagnetic mass shifts (or splittings) to order $\alpha$ and equal-time commutators is analyzed. If the invariants in the forward spin-averaged Compton amplitude go as $k^{-2}$ for $k^{2} \rightarrow \infty$, it is possible to relate the quadratic and logarithmic divergences in $\delta m^{2}$ to certain Lorentz scalars formed from the commutators. These results are illustrated by models with currents bilinear or linear in canonical fields. The Jost-Lehmann representation is then used to analyze more general behavior. It is shown that the spectral function may generate a higher-rank tensor character of the amplitude than that expected from its kinematic factors. The relation is then more complicated even if the commutators still exist. Finally, detailed criteria for the existence of equal-time commutators are developed.
\end{abstract}

\section{INTRODUCTION AND SUMMARY}

$W^{5}$ analyze here the following two problems: (1) The relation between divergences in electromagnetic mass shifts (and splittings) to order $\alpha$ of a particle and equal-time current commutators. In particular, we shall isolate the quantities governing the divergences, determine the general circumstances under which these quantities are related to equal-time commutators involving the currents and their time derivatives, and apply the results to a number of models of interest. (2) The requirements on the behavior of the forward Compton amplitude for the corresponding equal-time commutator matrix elements to exist, the analysis of which is based on the Jost-Lehmann representation of this amplitude. The successive results are summarized below.

We begin, in Sec. II, with the case in which the relevant invariant amplitudes in the forward spinaveraged Compton amplitude $M^{\mu \nu}(k, p)$ are sufficiently well behaved that the Bjorken prescription ${ }^{1}$ for the asymptotic behavior of the amplitude is justified. In that case, the quadratic and logarithmic divergences are seen to be related to the matrix elements of the equal-time commutators

$$
\begin{aligned}
C^{k}(\mathbf{r}) & \equiv\left\langle p\left|\left[j^{0}(\mathbf{r}, 0), j^{k}(0)\right]\right| p\right\rangle, \\
C^{k l}(\mathbf{r}) & \equiv\left\langle p\left|\left[\partial^{0} j^{k}(\mathbf{r}, 0)-\partial^{k} j^{0}(\mathbf{r}, 0), j^{l}(0)\right]\right| p\right\rangle,
\end{aligned}
$$

respectively. The relation is actually more involved in that it is not the matrix elements of these commutators, but rather those of certain related Lorentz scalar operators, which are the coefficients of the divergences. The mass shift, being a scalar, must be determined by such scalars, which are to be identified from the general forms of $C_{k}$ and $C_{k l}$.

* On leave of absence from Physics Department, University of Washington, Seattle, Wash.

† Work supported by U. S. Atomic Energy Commission under Grant No. RCO-1388B.

$\ddagger$ Work supported by U. S. Air Force Office of Aerospace Research under Office of Scientific Research Grant No. AF 368-67.

${ }^{1}$ J. D. Bjorken, Phys. Rev. 148, 1467 (1966).
Within this framework, the remainder of the section deals with a number of explicit models in which the currents are either bilinear in charged fields of various spins or linear in canonical fields. It is shown there that most of these models can be expected to lead to divergent shifts (or splittings).

In Sec. III we investigate the behavior of the amplitudes in a more general framework by use of the JostLehmann $^{2}$ representation, first developed for retarded commutators; we shall see that it is valid also for our time-ordered product as a result of the spectral conditions of the problem. The mass shift is now calculated in terms of the corresponding Jost-Lehmann spectral functions. If we assume that the amplitude as a whole is unsubtracted (otherwise there is a quartic divergence) and, for the moment, that the spectral functions are well behaved asymptotically as a function of the representation's mass variable, the quadratic mass shift takes a simple form. The latter is precisely that associated with the relevant scalar in the $\left[j^{0}, j^{l}\right]$ commutation, so that the Bjorken analysis result is still essentially valid. If the quadratic divergence then vanishes, the logarithmic one becomes relevant. A somewhat stronger assumption on the behavior of the spectral functions then enables us to show that the corresponding relation between the "scalar" part of $\left[\partial^{0} j^{k}-\partial^{k} j^{0}, j^{l}\right]$ and the logarithmic divergence also holds.

In Sec. IV we lift the assumptions on the JostLehmann spectral functions and look for the conditions under which the divergences of mass shifts are still related to the equal-time commutators. The first part of the question is then, of course, to find when the latter are well defined. In this general context, we define the unequal-time commutators as the boundary values of analytic functions, in the spirit of Wight$\operatorname{man}^{3}$; if the limit at equal times of this distribution in $x^{\mu}$ is also a distribution in $\mathbf{r}$, the equal-time commutators

\footnotetext{
${ }^{2}$ R. Jost and H. Lehmann, Nuovo Cimento 5, 1598 (1957).

3 A. S. Wightman, Phys. Rev. 101, 860 (1956).
} 
exist. The conditions for the commutators to exist are found in general; the asymptotic behavior of the invariant functions must be a sum of terms of the form $\left(1 / k^{2}\right)^{n}\left(\nu^{2} / k^{2}\right)^{m}$. To obtain the explicit forms it is necessary to know the number of subtractions in the Jost-Lehmann form; if there is at most one, it then follows that the existence of equal-time commutators depends on a single condition. The latter also guarantees that the commutators transform as components of a Lorentz tensor, of arbitrarily high rank, however. This complication, that the Lorentz transformation properties are not dictated by the explicit tensor structures in $\left\langle p\left|\left[j^{\mu}(x), j^{\nu}(0)\right]\right| p\right\rangle$ alone (i.e., by the transformation character of the $\left.j^{\mu}\right)$ means that a higher-rank tensor behavior may be generated by the spectral functions themselves. In that case the commutators still determine the divergences, but in a more complicated fashion, whereas the earlier direct relation holds if there are no higher-tensor properties.

The final section studies the implications of the above commutator existence conditions on the asymptotic behavior of the Jost-Lehmann representation. It is shown that the number of subtractions in the Jost-Lehmann representation for the time-ordered product is reduced as the existence condition removes an apparent logarithmic divergence in the representation.

Returning to the mass-shift question, there is, in general, a possible quartic divergence. For theories in which such terms are absent (and only these can be analyzed meaningfully), the coefficients of $k^{-2}$ and $k^{-4}$ in the amplitude are determined in terms of the Lorentz scalars in the $C^{k}$ and $C^{k l}$ equal-time commutators. Thus, wherever these equal-time commutators exist they do indeed determine, but in a generally complicated way through their associated Lorentz scalars, the quadratic and logarithmic divergences. ${ }^{3 \mathrm{a}}$ The quite general possible Lorentz structure of the commutators which was obtained also uncovers the complexity that may exist within these ostensibly simple "kinematical" relations. The underlying reason resides in the fundamental model dependence of the small distance properties of both equal-time commutators and mass shifts.

Finally, it may be of interest to summarize here our results on the lack of connection between subtractions in $\nu$ dispersion relations for the forward Compton amplitude and infinites of mass shifts or existence of

3a Note added in proof. It has recently been argued [R. A. Brandt and J. Sucher, Phys. Rev. Letters 20, 1131 (1968), and to be published] that the mass shift may be finite with nonvanishing equal-time commutators if the spectral function $\psi(s, \mathbf{u})$ oscillates. Their conclusion depends on the assertion that

$$
\int_{0}^{\infty} d x \cos x \equiv \lim _{n \rightarrow \infty} \int_{0}^{\infty} d x f_{n}(x) \cos x
$$

is undefined and can be assigned any value. However, it is clear (cf. the definition of the $\delta$ function) that this assertion can only be maintained by specifically choosing a set of testing functions $f_{n}(x)$ which always samples the same part of the cosine curve. Our contrary conclusions are in agreement with Epstein and Jackiw's ${ }^{22}$ analysis of the above work. equal-time commutators. We find that subtractions in $\nu$ are consistent with either finite or infinite mass shifts, while no subtractions do not exclude infinite shifts. Likewise, subtractions in $\nu$ are consistent with the existence of well-defined equal-time commutators, while no subtractions do not necessarily imply their existence, but only give bounds on the singularities of various commutators. These results follow from the relation of Jost-Lehmann to dispersion forms given in Sec. III.

\section{MASS-SHIFT DIVERGENCES IN SPECIFIC MODELS}

In this section we investigate the mass splittings in a number of general models under the assumption that the Bjorken limit ${ }^{1}$ holds. ${ }^{4} \mathrm{~A}$ critical discussion of the validity of this limit and of mass splittings in more general circumstances is given in subsequent sections. We recall Bjorken's prescription: The asymptotic behavior of the $k$ integral is determined by the limit $k_{0} \rightarrow \infty, \mathbf{k}$ fixed.

The invariant functions free of kinematic singularities are defined by

$$
\begin{aligned}
M^{\mu \nu}(p, k) \equiv & \left(k^{2} g^{\mu \nu}-k^{\mu} k^{\nu}\right) M_{1}\left(k^{2}, \nu\right)-\left(1 / m^{2}\right)\left[p^{\mu} p^{\nu} k^{2}\right. \\
& \left.-(p k)\left(p^{\mu} k^{\nu}+k^{\mu} p^{\nu}\right)+g^{\mu \nu}(p k)^{2}\right] M_{2}\left(k^{2}, \nu\right) \\
= & \int d x e^{-i k x} i\left\langle p\left|T^{*}\left(j^{\mu}(x) j^{\nu}(0)\right)\right| p\right\rangle,
\end{aligned}
$$

where $T^{*}$ represents the conserved Lorentz covariant current correlation function ${ }^{5}$ with the disconnected graphs removed. The single-particle states are covariantly normalized so that $\left\langle\mathbf{p} \mid \mathbf{p}^{\prime}\right\rangle=(2 \pi)^{3} 2 p^{0} \delta\left(\mathbf{p}-\mathbf{p}^{\prime}\right)$, we use the metric $(-1,1,1,1)$ throughout $\left(p^{2}+m^{2}=0\right)$, and $\nu \equiv-p k / m$.

The mass shift $\delta m^{2}$ may then be written in terms of $M^{\mu \nu} \mathrm{as}^{6}$

$$
\begin{aligned}
& \delta m^{2}=+\frac{1}{2} i e^{2}(2 \pi)^{-4} \int d^{4} k\left(k^{2}-i \epsilon\right)^{-1} M_{\mu}{ }^{\mu}(p, k) \\
&=+\frac{3}{2} i e^{2}(2 \pi)^{-4} \int d^{4} k\left(k^{2}-i \epsilon\right)^{-1} \\
& \\
& \quad \times\left[k^{2} M_{1}\left(k^{2}, \nu\right)+\frac{1}{3}\left(k^{2}-2 \nu^{2}\right) M_{2}\left(k^{2}, \nu\right)\right],
\end{aligned}
$$

where the conservation of the $T^{*}$ product has been used to retain only the $g_{\mu \nu}$ part of the photon propagator.

The quadratic and logarithmic divergences of the mass-shift calculation are determined by the coefficients

\footnotetext{
${ }^{4}$ Specific results for the mass splitting of nonsoft pions in the algebra of fields were obtained independently by the authors [S. Deser, Copenhagen Summer Institute Lecture 1967 (SINBI Proceedings, Copenhagen, 1967)]; M. Halpern and G. Segrè, Phys. Rev. Letters 19, 611 (1967); 19, 1000 (E) (1967); and G. C. Wick and B. Zumino, Phys. Letters 25, 479 (1967)

${ }^{5}$ See, for example, L. S. Brown, Phys. Rev. 150, 1338 (1966). 6 See, for example, W. N. Cottingham, Ann. Phys. (N.Y.) 25, 424 (1963).
} 
of $1 / k^{2}$ and $1 / k^{4}$, respectively, as $k^{2} \rightarrow \infty$ in the $M_{i}$. In order to make such an assertion, one must assume $M_{i}$ goes to zero at least as fast as $1 / k^{2}$; if that is not the case, the situation is much more complicated and the mass shift is more than quadratically divergent. We shall discuss this case in later sections.

The limit as $k^{2} \rightarrow \infty,\left|k^{2}\right| \gg|p k|$ is then, under the assumption that $M_{i} \sim\left(1 / k^{2}\right) m_{i}, m_{i}$ constant,

$$
\begin{gathered}
\lim _{k^{0} \rightarrow \infty} M^{\mu \nu}(p, k)=\left[\left(\left(g^{\mu \nu}+\delta_{0}{ }^{\mu} \delta_{0}{ }^{\nu}\right)+\frac{1}{k^{0}}\left(\delta_{0}^{\mu} k^{\nu}+k^{\mu} \delta_{0}{ }^{\nu}\right.\right.\right. \\
\left.\left.-2 \delta_{0}{ }^{\mu} \delta_{0}{ }^{\nu} k^{0}\right)\right) m_{1}-\frac{1}{m^{2}}\left(\left(p^{\mu} p^{\nu}-\delta_{0}{ }^{\mu} p^{0} p^{\nu}-p^{\mu} p^{0} \delta_{0}{ }^{\nu}-p^{02} g^{\mu \nu}\right)\right. \\
-\frac{1}{k^{0}}\left[p^{0}\left(k^{\mu} p^{\nu}+p^{\mu} k^{\nu}-\delta_{0}{ }^{\mu} k^{0} p^{\nu}-p^{\mu} k^{0} \delta_{0}{ }^{\nu}\right)-2 p^{0} \mathbf{p} \cdot \mathbf{k} g^{\mu \nu}\right. \\
\left.\left.\left.-\mathbf{p} \cdot \mathbf{k}\left(\delta_{0}^{\mu} p^{\nu}+p^{\mu} \delta_{0}{ }^{\nu}\right)\right]\right)\right],
\end{gathered}
$$

where the terms which go as $O(1)$ are just the Schwinger terms necessary to preserve the covariance of the amplitude. Consistent with the general structure of the current correlation function $M^{\mu \nu}$, these occur only in the space-space components. ${ }^{1,5}$ The $1 / k^{0}$ terms yield the equal-time commutators. The $m_{1}$ gives only a $\left[j^{0}, j^{k}\right]$ commutator, while $m_{2}$ yields a $\left[j^{k}, j^{l}\right]$ commutator as well. We recall that while the Schwinger terms occur in the $\left[j^{0}, j^{k}\right]$ commutators, these terms require that space-space parts be added to the timeordered product for Lorentz covariance.

On the other hand, the asymptotic behavior which we have assumed implies that the dominant term in the mass-shift calculation is

$$
\begin{aligned}
\delta m^{2} & \sim-\frac{3}{2} \frac{e^{2}}{(4 \pi)^{2}} \int^{\infty} k^{2} d k^{2}\left[\left(\frac{1}{k^{2}}\right) m_{1}+\frac{1}{2}\left(\frac{1}{k^{2}}\right) m_{2}\right] \\
& =-\frac{3 \alpha}{8 \pi} \int^{\infty} d k^{2}\left(m_{1}+\frac{1}{2} m_{2}\right),
\end{aligned}
$$

where the integral has been transformed to Euclidean space $^{6}$ and the angular integration performed.

The quadratic divergence is thus determined by $m_{1}$ and $m_{2}$ which are in turn determined by the matrix element of the $\left[j^{0}, j^{k}\right]$ commutator. The $c$-number parts of the commutator do not, of course, contribute since they are always subtracted along with the disconnected graphs.

The logarithmic divergences become interesting if, as in some models, the quadratic divergence vanishes. In that case, we assume that $M_{i} \sim \bar{m}^{i} / k^{4}$ and the asymptotic behavior as $k^{0} \rightarrow \infty$ is

$$
\begin{array}{r}
M^{\mu \nu} \underset{k^{0 \rightarrow \infty}}{\longrightarrow}\left(\frac{1}{k^{0}}\right)^{4}\left[\left(g^{\mu \nu} k^{2}-k^{\mu} k^{\nu}\right) \bar{m}_{1}-\left(1 / m^{2}\right)\left(p^{\mu} p^{\nu} k^{2}\right.\right. \\
\left.\left.-p k\left(p^{\mu} k^{\nu}+k^{\mu} p^{\nu}\right)+g^{\mu \nu}(p k)^{2}\right) \bar{m}_{2}\right],
\end{array}
$$

which is unrelated to any $\left[j^{\mu}(\mathbf{r}), j^{\nu}(0)\right]$. However if, we consider the curl of $j^{\sigma}, K^{\lambda \sigma} \equiv \partial^{\lambda} j^{\sigma}-\partial^{\sigma} j^{\lambda}$, this will introduce an additional factor of $k^{0}$, restoring the $1 / k^{0}$ behavior characteristic of equal-time commutators. Then

$$
\begin{aligned}
& \lim _{k^{0} \rightarrow \infty} \int d x e^{-i k x} i\left\langle p\left|T^{*}\left(K^{\lambda \sigma}(x), j^{\nu}(0)\right)\right| p\right\rangle \\
&=\lim _{k^{0} \rightarrow \infty} i\left\{\left(k^{\lambda} g^{\sigma \nu}-k^{\sigma} g^{\lambda \nu}\right) k^{2} M_{1}\left(k^{2},-p k\right)-\left(1 / m^{2}\right)\right. \\
& \times\left[\left(k^{\lambda} g^{\sigma \nu}-k^{\sigma} g^{\lambda \nu}\right)(p k)^{2}-\left(k^{\lambda} p^{\sigma}-p^{\lambda} k^{\sigma}\right)\right. \\
&\left.\left.\times\left(k^{\nu} p k-p^{\nu} k^{2}\right)\right] m_{2}\left(k^{2},-p k\right)\right\} \\
&=i\left[1 /\left(-k^{0}\right)\right]\left\{\left(\delta_{0}^{\lambda} g^{\sigma \nu}-\delta_{0}{ }^{\sigma} g^{\lambda \nu}\right) \bar{m}_{1}-\left(1 / m^{2}\right)\right. \\
& \times\left[p^{02}\left(\delta_{0}^{\lambda} g^{\sigma \nu}-\delta_{0}{ }^{\sigma} g^{\lambda \nu}\right)+\left(\delta_{0}{ }^{\alpha} p^{\sigma}-p^{\lambda} \delta_{0}{ }^{\sigma}\right)\right. \\
&\left.\left.\times\left(\delta_{0}{ }^{0} p^{0}-p^{\nu}\right)\right] \bar{m}_{2}\right\} .
\end{aligned}
$$

Note that there is no constant part this time, hence no noncovariant terms. The commutator is a $\delta$ function with an operator coefficient whose matrix element is given by the expression in curly brackets in the above equation. The only nonvanishing term is the commutator

$$
\begin{aligned}
C^{l k}(\mathbf{r}) & \equiv\left\langle p\left|\left[K^{0 l}(\mathbf{r}, 0), j^{k}(0)\right]\right| p\right\rangle \\
& \equiv i \delta(\mathbf{r})\left[\delta^{\delta k} \bar{m}_{1}+\left(1 / m^{2}\right)\left(\delta^{l k} p^{02}-p^{l} p^{k}\right) \bar{m}_{2}\right] \\
& \equiv i \delta(\mathbf{r}) \bar{C}^{00 t k} .
\end{aligned}
$$

The previous commutator $\left\langle p\left|\left[j^{0}, j^{l}\right]\right| p\right\rangle$ has the analogous form

$$
\begin{aligned}
C^{l}(\mathbf{r}) & \equiv i \partial_{k} \delta(\mathbf{r})\left[\delta^{l k} m_{1}+\left(1 / m^{2}\right)\left(\delta^{l k} p^{02}-p^{l} p^{k}\right) m_{2}\right] \\
& \equiv i \partial_{k} \delta(\mathbf{r}) C^{00 l k} .
\end{aligned}
$$

The commutator coefficients are just components of the fourth-rank tensors ${ }^{7} C^{\mu \nu \lambda \sigma}, \bar{C}^{\mu \nu \lambda \sigma}$ of the general form

$$
\begin{aligned}
& C^{\mu \nu \lambda \sigma}=-g^{\mu \nu} g^{\lambda \sigma} m_{1}+\left(1 / m^{2}\right)\left(p^{\mu} p^{\nu} g^{\lambda \sigma}+g^{\mu \nu} p^{\lambda} p^{\sigma}\right) m_{2} \\
& +\left(1 / m^{2}\right) p^{\mu} p^{\nu} g^{\lambda \sigma} m_{1}{ }^{(1)}+\alpha\left(g^{\mu \lambda} g^{\nu \sigma}+g^{\mu \sigma} g^{\nu \lambda}\right)+\beta\left(1 / m^{2}\right) \\
& \times\left(g^{\mu \lambda} p^{\nu} p^{\sigma}+g^{\mu \sigma} p^{\nu} p^{\lambda}+g^{\nu \lambda} p^{\mu} p^{\sigma}+g^{\nu \sigma} p^{\mu} p^{\lambda}\right) \text {. }
\end{aligned}
$$

Note that the $\alpha$ and $\beta$ coefficients have vanishing (00kl) components.

The most general fourth-rank tensor with the above symmetries contains an additional term of the form $p^{\mu} p^{\nu} p^{\lambda} p^{\sigma}$; we omit it since it is absent in the models to be discussed. It is shown that terms of the form $m_{1}{ }^{(1)}$ may occur and that the mass shift is determined by $m_{1}+\frac{1}{4} m_{1}{ }^{(1)}+\frac{1}{2} m_{2}$, but independent of $\alpha$ and $\beta$, as is the commutator (though such terms can and do arise in $C^{\mu \nu \lambda \sigma}$ itself, while a $p^{\mu} p^{\nu} p^{\lambda} p^{\sigma}$ term would contribute both to the commutator and the mass shift). We now find the appropriate Lorentz scalar part of $C^{\mu \nu \lambda \sigma}$ which determines this combination from the two independent

${ }^{7}$ Actually, the coefficients may have a more general structure involving higher than fourth-rank tensors. The correspondingly more complicated relation to $\delta m^{2}$ is discussed in Sec. IV. 


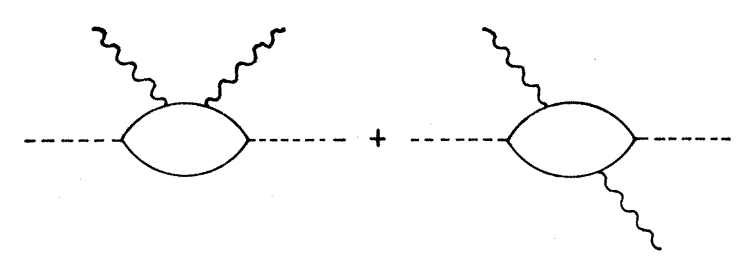

Fig. 1. Typical diagrams contributing to $M^{\mu \nu}$.

double traces

$$
\begin{aligned}
& C^{\mu}{ }_{\mu \nu}{ }_{\nu}=-16\left\{m_{1}+\frac{1}{4} m_{1}{ }^{(1)}+\frac{1}{2} m_{2}-\frac{1}{2} \alpha+\frac{1}{4} \beta\right\}, \\
& C^{\mu{ }_{\mu \nu}}=-4\left\{m_{1}+\frac{1}{4} m_{1}{ }^{(1)}+\frac{1}{2} m_{2}-5 \alpha+\frac{5}{2} \beta\right\} .
\end{aligned}
$$

Thus the coefficients of the quadratic and logarithmic mass shifts are given by, respectively,

and

$$
\begin{aligned}
& m_{1}+\frac{1}{4} m_{1}{ }^{(1)}+\frac{1}{2} m_{2}=(1 / 36)\left(C^{\mu \nu}{ }_{\mu \nu}-\frac{5}{2} C^{\mu}{ }_{\mu \nu}{ }^{\nu}\right) \\
& \bar{m}_{1}+\frac{1}{4} \bar{m}_{1}{ }^{(1)}+\frac{1}{2} \bar{m}_{2}=(1 / 36)\left(\bar{C}^{\mu \nu}{ }_{\mu \nu}-\frac{5}{2} \bar{C}^{\mu}{ }_{\mu \nu}{ }^{\nu}\right) .
\end{aligned}
$$

We now investigate the possibility of finite radiative corrections to mass shifts in some models. Internal symmetry as well as parity indices will be omitted throughout. The arguments then apply to mass shifts or splits, depending on which isospin parts are considered.

\section{A. Bilinear-Current Models (with Charged Bose Fields)}

Here the current is constructed bilinearly from charged fields which may be scalar, spinor, or vector. Then

$j^{\mu}=\psi^{\dagger} \alpha^{\mu} \psi+i\left(\varphi^{\dagger \mu} \varphi-\varphi^{\mu} \varphi^{\dagger}\right)+i\left(G^{\mu \nu} B_{\nu}-G^{\mu \nu} B_{\nu}^{\dagger}\right)$.

When there are no derivative couplings, the fields obey the canonical (anti) commutation relations:

$$
\begin{aligned}
\left\{\psi(\mathbf{r}), \psi^{\dagger}(0)\right\} & =\delta(\mathbf{r}), \\
{\left[\varphi^{\dagger 0}(\mathbf{r}), \varphi(0)\right] } & =i \delta(\mathbf{r}), \\
{\left[G^{0 k \dagger}(\mathbf{r}), B^{l}(0)\right] } & =i \delta^{k l} \delta(\mathbf{r}),
\end{aligned}
$$

and

$$
\begin{aligned}
\varphi^{k} & =\partial^{k} \varphi, \\
G^{k l} & =\partial^{k} B^{l}-\partial^{l} B^{k}, \\
B^{0} & =\left(1 / m^{2}\right)\left(g J^{0}-\partial_{l} G^{0 l}\right),
\end{aligned}
$$

where $J^{\mu}$ is the source of $B^{\mu}$ :

We also assume

$$
\partial_{\nu} G^{\mu \nu}+m^{2} B^{\mu}=g J^{\mu} \text {. }
$$

$$
\left[j^{0}(\mathbf{r}), J^{0}(0)\right]=-J^{0}(0) \delta(\mathbf{r}) .
$$

Explicit calculation yields

$$
\begin{aligned}
{\left[j^{0}(\mathbf{r}), j^{l}(0)\right] } & =i \partial_{k}\left\{\left[2 \varphi^{\dagger} \varphi \delta^{k l}+\left(1 / m^{2}\right)\left(G^{0 k \dagger} G^{0 l}+G^{0 l \dagger} G^{0 k}\right)\right.\right. \\
& \left.\left.+\delta^{k l} B^{\dagger m} B_{m}-B^{\dagger k} B^{l}-B^{\dagger l} B^{k}\right] \delta(\mathbf{r})\right\} .
\end{aligned}
$$

FIG. 2. Contribution to $\delta m^{2}$ for scalar meson

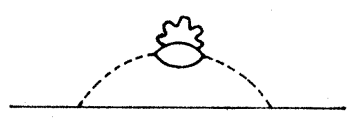

FIg. 3. Quadratically divergent contribution to pion mass shift.

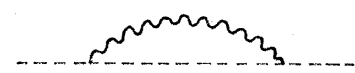

Here the absence of fermion terms just reflects the wellknown inconsistency in naive calculations of the equaltime commutator of fermion currents. We shall assume that this missing Schwinger term is a $c$ number and so will not contribute to the matrix elements in which we are interested. In a model where it is an operator it will, in general, contribute a quadratic divergence. In this connection we recall that the presence of other interactions may well affect a commutator's operator propperties. $^{8}$ As an example, consider the perturbation theory model of a neutral pseudoscalar meson interacting with charged nucleons through an interaction term of the form $g \varphi \bar{\psi} \gamma^{5} \psi$. The lowest-order diagrams for the current correlation function between meson states are of the forms shown in Fig. 1. The matrix element of the $\left[j^{0}, j^{k}\right]$ commutator can then be calculated and it does not vanish. Hence, the interactions have changed the commutator relations and the neutral meson will have quadratically divergent $\delta m^{2}$ in $O(\alpha)$. This will in turn entail a quadratic divergence in the nucleon through the $O(\alpha)$ term shown in the diagram of Fig. 2.

Although the above boson $\left[j^{0}, j^{k}\right]$ commutator is positive definite, the quantity which determines the mass shift is the difference between the diagonal matrix element and the vacuum expectation value, so there are no positive-definite conditions on the mass shift. While it is conceivable that just the diagonal matrix elements but not the operator vanish, thereby removing the quadratic divergence from the mass shifts, we know of no such local operator, except where kinematic constraints force it. This is clearly not the case here; thus we conclude that the general mass shift will be quadratically divergent in this model and must be absorbed into an explicit renormalization. It should perhaps be emphasized that, while most or all mass shifts will be divergent, this does not imply that there are an infinite number of independent renormalizations, but only that a shift of any mass will, in general, cause a shift of all other masses; hence a divergent mass shift of one mass will imply a similar divergence in all masses.

The formalism with which we are working is unable to disentangle the various sources of the divergence and a more detailed model is necessary to make any further statements. For a pion field coupled to a nucleon field, the pion has a quadratic divergence through the diagram of Fig. 3, and the nucleon then acquires a quadratic divergence through that of Fig. 4. In this model, once the pion has been renormalized, there are no further

FIG. 4. Quadratically divergent contribution to nucleon mass shift.

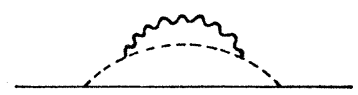

${ }^{8}$ K. A. Johnson and F. E. Low, Progr. Theoret. Phys. Suppl. 37 \& 38, 74 (1966). 
quadratic mass shifts but only shifts of the nucleon mass due to the (finite) shift of the renormalized pion masses.

The general fourth-rank tensor structure $C^{\mu \nu \lambda \sigma}$, which was defined earlier for the commutators, can now be explicitly exhibited for these models:

$$
\begin{aligned}
C^{\mu \nu \lambda \sigma}=-g^{\mu \nu} g^{\lambda \sigma}\left(2 \varphi^{\dagger} \varphi+B^{\dagger \mu} B_{\mu}\right)+\left(\frac{1}{2} m^{2}\right) \\
\times\left(G^{\mu \lambda \dagger} G^{\nu \sigma}+G^{\nu \lambda \dagger} G^{\mu \sigma}+G^{\mu \sigma \dagger} G^{\nu \lambda}+G^{\nu \sigma} G^{\mu \lambda}\right)-\frac{1}{2} g^{\lambda \sigma} \\
\quad \times\left(B^{\dagger \mu} B^{\nu}+B^{\dagger \nu} B^{\mu}\right)+g^{\mu \nu}\left(B^{\dagger \lambda} B^{\sigma}+B^{\dagger \sigma} B^{\lambda}\right) .
\end{aligned}
$$

The coefficient of the quadratic mass shift is then the matrix element of the operator

$$
\begin{aligned}
(1 / 36):\left(C^{\mu \nu}{ }_{\mu \nu}-\frac{5}{2} C^{\mu}{ }_{\mu \nu}{ }_{\nu}\right): \\
\quad=:\left[2 \varphi^{\dagger} \varphi+\frac{3}{4} B^{\dagger \mu} B_{\mu}-\left(1 / 6 m^{2}\right) G^{\dagger \mu \nu} G_{\mu \nu}\right]:
\end{aligned}
$$

\section{B. Bilinear-Current Models (without Charged Bose Fields)}

The logarithmic divergence can now be calculated from the commutator $\left[\partial^{0} j^{k}-\partial^{k} j^{0}, j^{l}\right]$; it becomes interesting only in the special case of a pure Fermi current $j^{\mu}=\bar{\psi} \gamma^{\mu} \psi$, where the quadratic divergence vanishes. The equations of motion for $\psi$ are defined to be

$$
\left(\alpha^{\mu} \partial_{\mu}+i m \beta\right) \psi=i \chi
$$

where $\chi$ is assumed to have the anticommutation relations

$$
\left\{\chi_{\alpha}(\mathbf{r}), \psi_{\beta}(0)\right\}=\varphi_{\alpha \beta}(0) \delta(\mathbf{r})
$$

and the components $\varphi_{\alpha \beta}$ transform like boson fields. Then

$$
\begin{aligned}
K^{0 l}=\partial^{0} j^{l}-\partial^{l} j^{0}=i \psi^{\dagger}(\sigma \times \overleftrightarrow{\nabla}) & -2 i m \psi^{\dagger} \alpha^{l} \psi \\
& -i\left(\psi^{\dagger} \alpha^{l} \chi-\chi^{\dagger} \alpha^{l} \psi\right)
\end{aligned}
$$

and

$$
\begin{array}{r}
{\left[K^{0 l}(\mathbf{r}), j^{k}(0)\right]=i \delta(\mathbf{r})\left\{\psi^{\dagger}\left[\boldsymbol{\alpha} \cdot(1 / i) \stackrel{\leftrightarrow}{\nabla} \delta^{k l}-\alpha^{l}(1 / i) \stackrel{\leftrightarrow}{\nabla^{k}}\right] \psi\right.} \\
\left.-4 m \bar{\psi} \psi \delta^{k l}-\psi^{\dagger} \alpha^{l} \varphi \alpha^{k} \psi-\psi^{\dagger} \alpha^{k} \varphi \alpha^{l} \psi+\chi^{\dagger} \alpha^{l} \alpha^{k} \psi+\psi^{\dagger} \alpha^{k} \alpha^{l} \chi\right\} \\
+i \epsilon^{0 l k p} \partial_{p} \delta(\mathbf{r}) \psi^{\dagger} \gamma^{5} \psi
\end{array}
$$

There are again no positive-definite statements concerning the vacuum-subtracted diagonal spin-averaged matrix elements to be made. The only form which does not contribute is the $\epsilon^{0 l k p} \partial_{p} \delta(\mathbf{r})$ term which has no scalar part and whose spin-averaged forward-scattering matrix element vanishes. The rest have nonvanishing matrix elements and we conclude, as above, that most mass shifts are logarithmically divergent, including those of any neutral bosons, due to the coupling between particles which was discussed in the case of quadratic divergence. We have not expressed the commutator in the $\vec{C}^{\mu \nu \lambda \sigma}$ form because a more explicit statement concerning the type of interaction is required for the $\bar{x} \psi$ and $\bar{\psi} \varphi \psi$ terms.

We have now exhausted the class of models for which we can consistently couple the electromagnetic field to a bilinear current and, in general, they all yield divergent mass shifts.

\section{Algebra of Fields}

Two further classes of electromagnetic couplings have been proposed which are, in some cases at least, more convergent than the previous ones. In the first ${ }^{9,10}$ the electromagnetic current is taken to be proportional to a canonical field,

$$
j^{\mu} \equiv \lambda \varphi^{\mu}, \quad G^{\mu \nu}=\partial^{\mu} \varphi^{\nu}-\partial^{\nu} \varphi^{\mu},
$$

where $G^{0 k}$ is canonically conjugate to $\varphi^{k}$.

The $\left[j^{0}, j^{k}\right]$ commutator is then a $c$ number,

$$
\lambda^{2}\left[\varphi^{0}(\mathbf{r}), \varphi^{k}(0)\right]=-i\left(\lambda^{2} / m^{2}\right) \partial^{k} \delta(\mathbf{r}) ;
$$

hence there are no quadratic divergences. Further,

$$
\left[\left(\partial^{0} j^{k}-\partial^{k} j^{0}\right)(\mathbf{r}), j^{l}(0)\right]=i \lambda^{2} \delta^{k l} \delta(\mathbf{r})
$$

is again a $c$ number and there are no logarithmic divergences either. Two versions of this class have been proposed. The first ${ }^{9}$ is inadequate as a description of the real world since the vector field is taken to be a linear combination of the $\rho, \varphi$, and $\omega$ fields. Then, either the vector field must have a Yang-Mills coupling, in which case the above analysis does not hold, or there must be additional explicit terms in the current corresponding to the current of the $\rho^{+}$and $\rho^{-}$and these bilinear boson terms produce the usual quadratic divergence. In the second ${ }^{10}$ the canonical field is taken to be an entirely new field which is coupled to what would normally be called the electromagnetic current. Then the above analysis does apply; however, the divergences reappear in the coupling of the new field.

When a Yang-Mills coupling is introduced, the equations must be modified to read

and

$$
G_{a}^{\mu \nu}=\partial^{\mu} \varphi_{a}^{\nu}-\partial^{\nu} \varphi_{a}^{\mu}-i g C_{a b c} \varphi_{b}^{\mu} \varphi_{c}^{\nu}
$$

so that

$$
\begin{gathered}
\partial_{\nu} G_{a}{ }^{\mu \nu}+m^{2} \varphi_{a}{ }^{\mu}=g \partial_{a^{\mu}} \\
j^{\mu}=\left(m^{2} / g\right) \varphi_{q^{\mu}},
\end{gathered}
$$

where $q$ is the component of the internal symmetry group corresponding to the charge. While

$$
\left[j^{0}(\mathbf{r}), j^{k}(0)\right]=-i\left(m^{2} / g^{2}\right) \partial^{k} \delta(\mathbf{r})
$$

is as before, the other commutator is altered since the curl of the $\varphi$ field is no longer the conjugate momentum to $j^{l}$ :

$$
\begin{aligned}
& \quad\left[\left(\partial^{0} j^{k}-\partial^{k} j^{0}\right)(\mathbf{r}), j^{l}(0)\right] \\
& \quad=\left(m^{2} / g\right)^{2}\left[G_{q}{ }^{k}(\mathbf{r})-i q \varphi_{a}{ }^{0}(\mathbf{r}) C_{a q b} \varphi_{b}^{k}(\mathbf{r}), \varphi_{q}{ }^{l}(0)\right] \\
& \quad=i\left(m^{2} / g\right)^{2} \delta(\mathbf{r})\left[\delta^{k l}+\left(g^{2} / m^{2}\right) \varphi_{a}^{k}\left(C_{q}\right)_{a b} \varphi_{b}{ }^{l}\right] .
\end{aligned}
$$

It should be emphasized that the indices of the structure constants include parity so that in the case of $S U(n) \otimes S U(n)$ we have a sum of vector and axialvector fields. The mass shifts must again be logarithmi-

${ }^{9}$ N. Kroll, T. D. Lee, and B. Zumino, Phys. Rev. 157, 1376 (1967).

${ }^{10}$ T. D. Lee, Phys. Rev. 168, 1714 (1968). 
cally divergent. The tensor $\tilde{C}^{\mu \nu \lambda \sigma}$ is readily identified as

$$
-\left(m^{2} / g\right)^{2} g^{\mu \nu}\left[g^{\lambda \sigma}+\left(g^{2} / m^{2}\right) \varphi_{a}{ }^{\lambda}\left(C_{q}^{2}\right)_{a b} \varphi_{b}{ }^{\sigma}\right]
$$

and the logarithmic mass divergence is proportional to the matrix element of

$$
\frac{1}{4} m^{2}: \varphi_{\mu a}\left(C_{q}\right)^{2}{ }_{a b} \varphi_{b}{ }^{\mu}: .
$$

It is perhaps worth noting that this is the only operator relevant to the logarithmic mass-shift divergences. The matrix elements of this operator could vanish without : $\varphi^{k} C_{q}{ }^{2} \varphi^{l}$ : vanishing-the latter is not equivalent to $: \varphi_{\mu} C_{q}{ }^{2} \varphi^{\mu}:$ in any one frame although it has been suggested as a test for convergence. ${ }^{4}$ In this model the vanishing of $\left\langle p\left|: \varphi_{\mu} C_{q}{ }^{2} \varphi^{\mu}:\right| p\right\rangle$ is the content of the general requirement

$$
m_{1}+\frac{1}{2} m_{2}+\frac{1}{4} m_{1}{ }^{(1)}=\left\langle p\left|(1 / 36)\left(C^{\mu \nu}{ }_{\mu \nu}-\frac{5}{2} C^{\mu} \mu_{\mu \nu}{ }_{\nu}\right)\right| p\right\rangle=0
$$

for the shifts to vanish. On the other hand, there is no single frame in which the vanishing of $\left\langle p\left|C^{00 k l}\right| p\right\rangle$ is sufficient; furthermore, the requirement that this element vanishes in all frames is stronger than is needed, so that vanishing divergences could conceivably occur with

$$
\left\langle p\left|(1 / 36)\left(C^{\mu \nu}{ }_{\mu \nu}-\frac{5}{2} C^{\mu}{ }_{\mu \nu}{ }_{\nu}\right)\right| p\right\rangle=0,\left\langle p\left|C^{00 k l}\right| p\right\rangle \neq 0
$$

for arbitrary $\mathbf{p}$.

In view of the well-known finite mass shift results obtained from $S U_{2} \times S U_{2}$ for soft pions ${ }^{11}$ using the Weinberg sum rules, ${ }^{12}$ it may be amusing to see how these are recovered from our general criteria. Here the quadratic divergence of the mass split vanishes, being governed by

$$
\left\langle\pi^{+}\left|\left[j^{0}, j^{k}\right]\right| \pi^{+}\right\rangle-\left\langle\pi^{0}\left|\left[j^{0}, j^{k}\right]\right| \pi^{0}\right\rangle,
$$

since the commutator is a $c$ number. The logarithmic divergence for the shift which includes both $I=0$ and $=2$ parts is governed by

$$
\begin{aligned}
\left\langle\pi^{ \pm}\right| & \left(\left\{V_{\mu}^{+}, V^{\mu-}\right\}+\left\{A_{\mu}^{+}, A^{\mu-}\right\}\right)\left|\pi^{ \pm}\right\rangle \\
= & \langle 0|\left(\left[Q_{5}{ }^{\mp},\left[\left\{V_{\mu}^{+}, V^{\nu-}\right\}+\left\{A_{\mu}^{+}, A^{\nu-}\right\}, Q_{5} \pm\right]\right]|0\rangle\right. \\
= & \left\langle 0\left|\left[Q_{5}{ }^{\mp}, \mp\left\{V_{\mu}^{ \pm}, A^{\mu 3}\right\} \mp\left\{A_{\mu}^{ \pm}, V^{\mu 3}\right\}\right]\right| 0\right\rangle \\
= & \langle 0|\left(\left\{A_{\mu}^{3}, A^{\mu 3}\right\}-\left\{V_{\mu}^{+}, V^{\mu-}\right\}+\left\{V_{\mu}^{3}, V^{\mu 3}\right\}\right. \\
& \left.\quad-\left\{A_{\mu}^{+}, A^{\mu-}\right\}\right)|0\rangle=0
\end{aligned}
$$

and vanishes by isospin conservation. Here we have reduced the soft pions by the axial charges $Q_{5}{ }^{ \pm}$. The divergent neutral-pion shift also vanishes in the same way. Direct use of the field algebra has bypassed explicit use of the sum in evaluating the divergent terms. The critical role of the soft-pion property was of course to reduce the general diagonal matrix element to a vacuum expectation value.

\section{JOST-LEHMANN REPRESENTATION}

In the previous section we have been considering the covariant time-ordered product, which appears directly

\footnotetext{
${ }^{11} \mathrm{~T}$. Das et al., Phys. Rev. Letters 18, 759 (1967).
}

${ }^{12}$ S. Weinberg, Phys. Rev. Letters 18, 507 (1967). in the expression for the mass shift and assuming the conditions needed for the Bjorken limit to be valid. We must now investigate the general behavior of the amplitude more carefully. For this purpose we turn to an explicit parametrization of the causal commutator-the Jost-Lehmann ${ }^{2}$ representation. Because of the particular mass conditions in the matrix elements of interest here, there is no necessity to go to the more general Dyson form and we can explicitly write, in the rest frame of $p^{\mu}$,

$$
\begin{gathered}
\left\langle p\left|\left[j^{\mu}(x), j^{\nu}(0)\right]\right| p\right\rangle=\int \frac{d k}{(2 \pi)^{3}} e^{i k x} M_{c}^{\mu \nu}(p, k), \\
M_{c}^{\mu \nu}(p, k)=\int_{0}^{\infty} d s \int d^{3} u \epsilon\left(k^{0}\right) \delta\left(s+|\mathbf{u}|^{2}+k^{2}-2 \mathbf{u} \cdot \mathbf{k}\right) \\
\times\left\{\left(g^{\mu \nu} k^{2}-k^{\mu} k^{\nu}\right) \Psi_{1}(s,|\mathbf{u}|)-\left(1 / m^{2}\right)\left[p^{\mu} p^{\nu} k^{2}\right.\right. \\
\left.\left.-(p k)\left(p^{\mu} k^{\nu}+k^{\mu} p^{\nu}\right)+g^{\mu \nu}(p k)^{2}\right] \Psi_{2}(s,|\mathbf{u}|)\right\} .
\end{gathered}
$$

Owing to crossing (antisymmetry under $\mu \leftrightarrow \nu, k \leftrightarrow-k$ ), the usual odd terms in $k_{0}$ do not appear. Rotational invariance and the fact that a spin average has been taken implies that the $\Psi_{i}$ depend only on $|\mathbf{u}|$ rather than $\mathbf{u}$. Note that while the Jost-Lehmann representation was originally written in the rest frame of the external particle, a straightforward Lorentz transformation gives it in the general frame $\mathbf{p} \neq 0$; there $\nu=k^{0}=-p k / m$ and $|\mathbf{k}|=\left(k^{2}+k^{02}\right)^{1 / 2}=\left(k^{2}+\nu^{2}\right)^{1 / 2}$.

In general, it is not possible to infer the form of the $T$ product by first obtaining the form of the two orderings $\left\langle p\left|j^{\mu} j^{\nu}\right| p\right\rangle$ and $\left\langle p\left|j^{\nu} j^{\mu}\right| p\right\rangle$ from the causal commutator, since the spectra of the two orderings overlap; however, in our case, (as long as the particle is stable under the strong interactions) the lowest intermediate state mass is the same as the external one. Thus for $j^{\mu} j^{\nu}$ the lowest value of $k^{0}$ is $\left(\mathbf{k}^{2}+m^{2}\right)^{1 / 2}-m \geq 0$. For the other order we have $k^{0} \leq m-\left(\mathbf{k}^{2}+m^{2}\right)^{1 / 2} \leq 0$ and the two orderings are distinguished by the sign of their frequencies:

$$
\begin{aligned}
& \left\langle p\left|j^{\mu}(x) j^{\nu}(0)\right| p\right\rangle=\int \frac{d k}{(2 \pi)^{3}} e^{i k x} M_{+}{ }^{\mu \nu}(p, k), \\
& \left\langle p\left|j^{\nu}(0) j^{\mu}(x)\right| p\right\rangle=\int \frac{d k}{(2 \pi)^{3}} e^{i k x} M_{-}{ }^{\mu \nu}(p, k),
\end{aligned}
$$

where

$$
\begin{gathered}
M_{ \pm}^{\mu \nu}(p, k)=\int_{0}^{\infty} d s \int d^{3} u \theta\left( \pm k^{0}\right) \delta\left(s+(\mathbf{u}-\mathbf{k})^{2}-k^{02}\right) \\
\times\left\{\left[g^{\mu \nu} k^{2}-k^{\mu} k^{\nu}\right] \Psi_{1}(s,|\mathbf{u}|)-\left(1 / m^{2}\right)\left[p^{\mu} p^{\nu} k^{2}\right.\right. \\
\left.\left.-(p k)\left(p^{\mu} k^{\nu}+k^{\mu} p^{\nu}\right)+g^{\mu \nu}(p k)^{2}\right] \Psi_{2}(s,|\mathbf{u}|)\right\} .
\end{gathered}
$$

We can now calculate the (usual) time-ordered product

$$
i\left\langle p\left|T\left(j^{\mu}(x) j^{\nu}(0)\right)\right| p\right\rangle=\int \frac{d k}{(2 \pi)^{4}} e^{i k x} \bar{M}^{\mu \nu}(p, k),
$$


where

$$
\begin{aligned}
& \bar{M}^{\mu \nu}(p, k)+\delta^{m n} \int d s d^{3} u \Psi_{1}(s,|\mathbf{u}|)+\left(\delta^{m n} p^{02}-p^{m} p^{n}\right) \\
& \times \int d s d^{3} u \Psi_{2}(s,|\mathbf{u}|)=\int_{0}^{\infty} d s \int \frac{d^{3} u}{s+(\mathbf{u}-\mathbf{k})^{2}-k^{02}-i \epsilon} \\
& \quad \times\left\{\left(g^{\mu \nu} k^{2}-k^{\mu} k^{\nu}\right) \Psi_{1}(s,|\mathbf{u}|)-\left(1 / m^{2}\right)\left[p^{\mu} p^{\nu} k^{2}\right.\right. \\
& \left.\left.\quad-(p k)\left(p^{\mu} k^{\nu}+k^{\mu} p^{\nu}\right)+g^{\mu \nu}(p k)^{2}\right] \Psi_{2}(s,|\mathbf{u}|)\right\}
\end{aligned}
$$

In general the $k^{0}$ s appearing in the kinematical tensor structures will give rise to noncovariant terms, reflecting the general lack of covariance of timeordered products when the equal-time commutators contain gradients of $\delta$ functions. ${ }^{13}$ The addition of "seagull" terms is required to obtain a covariant conserved current correlation function (for consistent coupling to the electromagnetic field).

Another problem which may arise is a lack of convergence of the $s$ integral in $M_{i}$. If that occurs, subtractions must be made and the subtraction constants correspond to additional interaction of the electromagnetic field with the matter fields. These represent additional parameters describing the interaction of photons with the charged particles involved, hence a redefinition of the electromagnetic interaction. We assume that some choice of subtraction constants results in the correct Compton scattering and mass shifts and investigate the consequences of such forms for the current correlation function. The degree of divergence of $\delta m^{2}$ is closely tied to the asymptotic behavior assumed for the Jost-Lehmann spectral function, i.e., to the number of subtractions required. Physically, the structure of the covariants associated with one subtraction corresponds to counter terms, in a simple Lagrangian picture, of forms like $\sim F_{\mu \nu} F^{\mu \nu} \phi^{2}$. Such an $O\left(k^{2}\right)$ momentum dependence generates a quartic divergence. Similarly, a still more rapid increase of $\Psi$ associated with, say, two subtractions, would lead to $\sim \Lambda^{6}$ divergence through counter terms of the form $\sim F_{\mu \nu} F^{\mu \nu} \partial^{2} \phi^{2}$ with $O\left(k^{4}\right)$ dependence. It may perhaps be mentioned in this context that we have treated all the usual constraints in the theory, such as the bare mass, as finite, i.e., that the usual renormalizations are finite; as a result normal counter terms like $\left(\delta m^{2}\right) \phi^{2}$ did not have to be worried about in this context. If, on the other hand, we were to take seriously this type of infinity, it would strongly alter the effective structure

\footnotetext{
${ }^{13}$ The derivation given here is not rigorous since it does not allow for the possibility of any further noncovariance of the time-ordered product arising from further derivatives of the delta functions in the commutator; but the Jost-Lehmann form is general enough to admit an arbitrary number of derivatives of the $\delta$ function. The final result has already included the subtractions which are necessary in that case; we shall not pursue the question further except to observe that the final expression (3.3) is covariant and has the correct analyticity properties, hence we choose it as our amplitude; as we shall see below, the amplitude is not completely defined when such derivatives are present.
}

of the theory. ${ }^{14}$ For the remainder of this section we shall assume that the current correlation function obeys a once-subtracted Jost-Lehmann representation. ${ }^{15}$

Provided that the spectral function $\Psi(s, u)$ is sufficiently regular at $u=0$,

$$
\lim _{\epsilon \rightarrow 0} \int_{|\mathbf{u}|<\epsilon} d^{3} u f(u) \Psi(s,|\mathbf{u}|)=0,
$$

a dispersion relation in $\nu^{2}$ can be derived. If we assume that the Jost-Lehmann representation is once subtracted, the once-subtracted relation reads, subtracting at $\mathbf{k}=0, k^{0}=i a,{ }^{16}$

$$
\begin{aligned}
& M_{i}\left(k^{2}, \nu\right)-M_{i}\left(a^{2}, i a\right) \\
& =\int d^{3} u d s \Psi_{i}(s,|\mathbf{u}|)\left(\frac{1}{s+(\mathbf{u}-\mathbf{k})^{2}-k^{02}-i \epsilon}\right. \\
& =4 \pi \int_{0}^{\infty} u^{2} d u d s \Psi_{i}(s, u)\left(\frac{1}{s+|\mathbf{u}|^{2}+a^{2}}\right) \\
& \left.\quad \times \ln \frac{s+(u+|\mathbf{k}|)^{2}-k^{02}-i \epsilon}{s+(u-|\mathbf{k}|)^{2}-k^{02}-i \epsilon}-\frac{1}{s+u^{2}+a^{2}}\right)
\end{aligned}
$$

This may be cast into a dispersion form by the following steps: Define

$$
I \Psi_{i}(s, u) \equiv \int_{0}^{s} d s^{\prime} \Psi_{i}\left(s^{\prime}, u\right)
$$

and make a Lorentz transformation to an arbitrary frame

$$
\begin{aligned}
M_{i}\left(k^{2}, \nu\right)- & M_{i}\left(a^{2}, i a\right)=4 \pi \int_{0}^{\infty} d s u^{2} d u I \Psi_{i}(s, u) \\
& \times\left(\frac{1}{\left(s+u^{2}+k^{2}\right)^{2}-4 u^{2}\left(\nu^{2}+k^{2}\right)}-\frac{1}{\left(s+u^{2}+a^{2}\right)^{2}}\right),
\end{aligned}
$$

where $\nu=-p k / m,|\mathbf{k}|=\left(k^{2}+\nu^{2}\right)^{1 / 2}$. The $(s, u)$ plane integration may then be rewritten as

$$
\begin{aligned}
& M_{i}\left(k^{2}, \nu\right)-M_{i}\left(a^{2}, i a\right) \\
& =4 \pi \int_{0}^{\infty} \frac{\nu^{\prime} d \nu^{\prime}}{\left(\nu^{\prime 2}+k^{2}\right)^{1 / 2}} \int_{\left(\nu^{\prime 2}+k^{2}\right)^{\frac{1}{2}-\nu^{\prime}}}^{\left(\nu^{\prime 2}+k^{2}\right)^{\frac{1}{2}+\nu^{\prime}}} \frac{1}{2} u d u \\
& \quad \times I \Psi_{i}\left(-u^{2}-k^{2}+2 u\left(\nu^{\prime 2}+k^{2}\right)^{1 / 2}, u\right) \\
& \quad \times\left(\frac{1}{\nu^{\prime 2}-\nu^{2}}-\frac{1}{\left[\left(\nu^{\prime 2}+k^{2}\right)^{1 / 2}-\left(k^{2}-a^{2}\right) / 2 u\right]^{2}}\right) .
\end{aligned}
$$

${ }^{14}$ This essential problem, which we have avoided, is discussed by W. Zimmerman, New York University report (unpublished). ${ }_{15}$ The above representation differs from that of Jost-Lehmann in that it is for a $T$ product rather than a retarded commutator; that it is a valid representation follows from the spectral conditions just discussed.

${ }_{16}$ Note that the subtraction point is specified by the full $k_{\mu}$ rather than by a single number owing to the explicit dependence of the Jost-Lehmann denominator on $k_{\mu}$. 
The last equation was obtained, for $k^{2}>0$, by the change of variables $s=-u^{2}-k^{2}+2 u\left(\nu^{\prime 2}+k^{2}\right)^{1 / 2}$; the limits are just such as to guarantee that $s>0$. The $u$ integral is over a finite region since $\Psi_{i}$ vanishes for $u>m$. If there is a finite contribution from $\mathbf{u}=0$, it has been dropped, since the integral explicitly does not include that point; a more careful treatment shows that inclusion of the point then yields a subtraction in $\nu^{2}$. Thus, an unsubtracted Jost-Lehmann representation does not imply an unsubtracted dispersion relation in $\nu$, unless $\Psi_{i}$ is also regular at $\mathbf{u}=0$.

We are now in a position to discuss the relation between subtractions in $\nu$ and the validity of the Bjorken analysis. The equal-time commutators may all exist and the asymptotic limit as $k_{0} \rightarrow \infty, \mathbf{k}$ finite, would then exist and be well behaved (the exact forms are discussed below). This, in itself, says nothing concerning the behavior in $\nu$ for fixed $k^{2}$. Thus, it is quite possible to have subtractions in $\nu$ and well-behaved current commutators with finite mass shifts. ${ }^{17}$

On the other hand, if an unsubtracted dispersion relation in $\nu$ is assumed, the situation is somewhat more complicated. This does not in itself guarantee the existence of the commutators, although it does imply that the Jost-Lehmann representation is unsubtracted (subtractions from the high-s behavior would be polynomials in $k$, while those from the $u=0$ singularities would be analytic functions of $k^{2}$ with cuts from $k^{2}=0$ to $-\infty$, hence they could not cancel). Also, it is not possible to deduce the behavior of $M\left(k^{2}, \nu\right)$ for large $k^{2}$ and $\nu^{2} / k^{2}$ finite, given that it is unsubtracted in $\nu$; it is necessary to make the additional assumptions that, in this limit, $M$ is independent of $\nu$ and is a polynomial in $1 / k^{2}$ to draw the conclusions of Ref. 1 .

The task now before us is to recast the mass shift in terms of the spectral functions $\Psi_{i}(s, u)$ rather than in terms of the time-ordered product. The convergence properties of the shifts will then be discussed on the basis of the spectral functions. Going to the Euclidean integral, we have

$$
\begin{gathered}
\delta m^{2}=-\frac{3}{2} e^{2} \int \frac{d^{4} k}{(2 \pi)^{4}}\left[M_{1}\left(k^{2}, i k_{4}\right)+\frac{1}{3}\left(1+\frac{2 k_{4}^{2}}{k^{2}}\right) M_{2}\left(k^{2}, i k_{4}\right)\right] \\
=-(3 \alpha / 4 \pi) \int d s d^{3} u \frac{d^{4} k}{2 \pi^{2}} \\
\quad \times\left[\Psi_{1}(s, u)+\frac{1}{3}\left(1+\frac{2 k_{4}^{2}}{k^{2}}\right) \Psi_{2}(s, u)\right] \\
\quad \times\left(\frac{1}{s+(\mathbf{u}-\mathbf{k})^{2}+k_{4}^{2}}-\frac{1}{s+a^{2}+u^{2}}\right)
\end{gathered}
$$

${ }^{17}$ There have been attempts to discuss the convergence of electromagnetic mass splittings on the basis of a Regge analysis of the amplitude $M^{\mu \nu}$ [H. Harari, Phys. Rev. Letters 17, 1303 (1966); W. N. Cottingham and J. Gibb, ibid. 18, 833 (1967)]. However, we have just seen that the Regge asymptotic behavior (in $\nu$ ) is not that which determines the convergence. In the second plus the subtraction constants, if any, which would give quartic divergences. We shall assume that there is no net quartic divergence, since otherwise the following considerations become somewhat academic. (Also, the transformation to the Euclidean integral is no longer valid if the amplitude is subtracted.)

The angular integral in $k$ can easily be performed:

$$
\begin{aligned}
& \delta m^{2}=-(3 \alpha / 4 \pi) \int_{0}^{\infty} \frac{1}{2} k^{2} d k^{2} d s d^{3} u \\
& \times\left\{\Psi_{1}(s, u)\left(\frac{2}{s+u^{2}+k^{2}+\gamma}-\frac{1}{s+u^{2}+a^{2}}\right)+\frac{1}{3} \Psi_{2}(s, u)\right. \\
& \times\left[\left(1+\frac{1 s+u^{2}+k^{2}+2 \gamma}{3}\right) \frac{2}{s+u^{2}+k^{2}+\gamma}\right) \\
& -\frac{3}{2+u^{2}+k^{2}+\gamma} \\
&
\end{aligned}
$$

where

$$
\gamma=\left[\left(s+u^{2}+k^{2}\right)^{2}-4 u^{2} k^{2}\right]^{1 / 2} .
$$

This expression will be used later in the general case; for the moment we shall consider it only in the case where no subtractions are needed and for the remainder of this section proceed under the even stronger assumption that the $s$ integral converges independently of $u$. For, as we are about to show, these assumptions are sufficient for the validity of the Bjorken arguments as applied to the quadratic divergences in the previous section. The additional assumptions for the corresponding logarithmic terms will be developed later. The asymptotic behavior in $k^{2}$ of the integrand is then simply obtained by replacing the integrand of the $s, u$ integral by its asymptotic value as a function of $k^{2}$. The quadratic mass shift becomes, upon expanding in $k^{-2}$,

$$
\begin{aligned}
\delta m^{2} \sim-\left(\frac{3 \alpha}{4 \pi}\right)^{1 / 2} \int^{\infty} d k^{2} \int d^{3} u d s \\
\times\left[\Psi_{1}(s, u)+\frac{1}{2} \Psi_{2}(s, u)\right]
\end{aligned}
$$

and dropping less singular terms. Its dependence on the spectral functions is thus through the particular combination $\left(\Psi_{1}+\frac{1}{2} \Psi_{2}\right)$.

We next also express the equal-time commutator $\left\langle p\left|\left[j^{0}, j^{k}\right]\right| p\right\rangle$ in terms of the $\Psi_{i}$. The relations thus obtained between the mass shift and the commutator will establish the equivalence of the above equation and the commutator forms of the previous section. For, in terms of its definition, we find for the commutator

$$
\begin{array}{r}
\left\langle p\left|\left[j^{0}(\mathbf{r}), j^{k}(0)\right]\right| p\right\rangle=i \partial_{l} \delta(\mathbf{r})\left(\delta^{k l} \int d s d^{3} u \Psi_{1}(s, u)\right. \\
\left.+\frac{1}{m^{2}}\left(\delta^{k l} p^{02}-p^{k} p^{l}\right) \int d s d^{3} u \Psi_{2}(s, u)\right) .
\end{array}
$$

paper, the authors attempt an analysis similar to ours, but neglect the possibility of a finite contribution from $\mathbf{u}=0$ and are therefore unable to give explicit conditions for convergence. 
The right-hand side has exactly the structure of $C^{00 k l}$ with $m_{1}(1)=0$, so that we may identify the $m_{i}$ in Sec. II as $\int d s d^{3} u \Psi_{i}(s, u)$ given earlier, thereby verifying that, under the stated conditions on the $\Psi_{i}$, the relation between the equal-time commutator and the shift still holds.

If the further assumption is made that the relevant scalar part of the $\left[j^{0}, j^{k}\right]$ commutator vanishes, the logarithmic divergence becomes dominant. However, its determination appears quite complicated unless the entire $\left[j^{0}, j^{k}\right]$ commutator vanishes, i.e., unless the conditions $\int d^{3} u d s \Psi_{i}(s, u)=0$ hold separately. With the further assumption that $\int d s \Psi_{i}(s, u)=0$, rather than just its $u$ integral, we obtain the result

$$
\begin{aligned}
\delta m^{2} \sim-\left(\frac{3 \alpha}{4 \pi}\right)^{1 / 2} \int^{\infty} \frac{d k^{2}}{k^{2}} \int d^{3} u d s(-s) \\
\times\left[\Psi_{1}(s, u)+\frac{1}{2} \Psi_{2}(s, u)\right],
\end{aligned}
$$

relating the logarithmic divergence to the first $s$ moment of $\Psi_{1}+\frac{1}{2} \Psi_{2}$. As in the above discussion of the quadratic parts, we now show that the same combination occurs in the corresponding commutator $\left\langle p\left|\left[K^{0 l}(\mathbf{r}), j^{k}(0)\right]\right| p\right\rangle$. The curl of the current commutator expression, Eq. (3.1), is

$$
\begin{aligned}
& \left\langle p\left|\left[K^{\mu \nu}(x), j^{\lambda}(0)\right]\right| p\right\rangle \\
& \quad=i \int \frac{d^{4} k}{(2 \pi)^{4}} e^{i k x}\left(k^{\mu} M_{c}{ }^{\nu \lambda}-k^{\nu} M_{c}{ }^{\mu \nu}\right)(p, k),
\end{aligned}
$$

whose only nonvanishing component at equal times reduces to

$$
\begin{aligned}
&\left\langle p\left|\left[K^{0 l}(\mathbf{r}), j^{k}(0)\right]\right| p\right\rangle \\
&=i \delta(\mathbf{r})\left(\delta^{k l} \int d s d^{3} u(-s) \Psi_{1}(s, u)+\frac{1}{m^{2}}\left(\delta^{k l} p^{02}-p^{k} p^{l}\right)\right. \\
&\left.\quad \times \int d^{3} u d s(-s) \Psi_{2}(s, u)\right)
\end{aligned}
$$

The quantities $\bar{m}_{i}$ can be identified as $\int d s d^{3} u(-s)$ $\times \Psi_{i}(s, u)$ and the analysis now clearly goes through just as in the quadratic case, to justify the simple mass-shift commutator relation for the logarithmic divergence (at least when $\left\langle p\left|\left[j^{0}, j^{l}\right]\right| p\right\rangle$ vanishes and the further assumptions made on $\Psi_{i}$ hold).

\section{CONDITIONS FOR EXISTENCE OF COMMUTATORS}

In the preceding sections we have made rather strong assumptions concerning the behavior of the
Jost-Lehmann spectral functions in order to obtain simple forms and relations for leading divergences and equal-time commutators. There are two major questions to be discussed in the general case: (i) How are the equal-time commutators expressed in terms of the (more general) spectral functions and what conditions must be obeyed by the latter for the commutators to be at all defined? (ii) Under what conditions are the mass shifts and equal time commutators related? A third question-what determines the mass shifts when the commutators do not exist?-can also be answered, but the result is not very illuminating. We shall start with the requirements for the equal-time commutators to exist. When they do and, further, have the Lorentz transformation properties which are dictated by the kinematical factors, the analysis of the previous section holds in its entirety. On the other hand, more general structures may occur, in which case knowledge of the equal-time commutators still allows the calculation of the divergence but the relation is no longer so simple.

The first problem, then, is to give a general definition of the equal-time commutators. One approach ${ }^{18}$ is to recognize that the operators involved are distributionvalued and that only in special cases can one consider them as defined at given times; thus in the more general case, one should integrate with a suitable testing function in time and then consider the limit in which these functions are localized at $t=0$.

An alternative approach, which we shall adopt, is to begin with the Wightman product as the boundary value of an analytic function. ${ }^{3}$ Thus

$$
\begin{aligned}
& \left\langle p\left|j^{\mu}(x) j^{\nu}(0)\right| p\right\rangle=e^{-i p x} F_{+}{ }^{\mu \nu}(x, p), \\
& \left\langle p\left|j^{\nu}(0) j^{\mu}(x)\right| p\right\rangle=e^{i p x} F_{-}{ }^{\mu \nu}(x, p),
\end{aligned}
$$

where $F_{ \pm}{ }^{\mu \nu}(z, p)$ is analytic in the cone $z^{\mu}=x^{\mu} \mp i \eta^{\mu}$, $\eta_{\mu} \eta^{\mu}<0, \eta^{0}>0$. The functions $F_{ \pm}{ }^{\mu \nu}(x, p)$ are the limit as $\eta \rightarrow 0$ within the cone.

We then define the commutator by

$$
\begin{aligned}
\lim _{\eta \rightarrow 0}\left\{e^{i p x} F_{+}{ }^{\mu \nu}(x-i \eta, p)-e^{i p x} F_{-}{ }^{\mu \nu}(x+i \eta, p)\right\} \\
\equiv\left\langle p\left|\left[j^{\mu}(x), j^{\nu}(0)\right]\right| p\right\rangle .
\end{aligned}
$$

This, of course, yields a distribution in $x^{\mu}$, which may or may not exist as a distribution in $\mathbf{r}$ at $t=0$. If it does, then the equal-time commutator exists and we obtain the same result as the time-distribution approach; if not, then the equal-time commutator does not exist as such and we shall not attempt to force a definition any further than to identify the various singularities as $\eta \rightarrow 0$.

${ }^{18}$ B. Schroer and P. Stichel, Commun. Math. Phys. 3, 258 (1966). 
In terms of our spectral functions, the Wightman where product is given by

$$
\begin{aligned}
& \left\langle p\left|j^{\mu}(x-i \eta) j^{\nu}(0)\right| p\right\rangle \\
& =\left(\partial^{\mu} \partial^{\nu}-g^{\mu \nu} \partial^{2}\right) \int_{0}^{\infty} d s \psi_{1}\left(s, \xi^{2}\right) \Delta^{(+)}(x-i \eta, s) \\
& +\frac{1}{m^{2}}\left[p^{\mu} p^{\nu} \partial^{2}-p \partial\left(p^{\mu} \partial^{\nu}+\partial^{\mu} p^{\nu}\right)+g^{\mu \nu}(p \partial)^{2}\right] \\
& \quad \times \int_{0}^{\infty} d s \psi_{2}\left(s, \xi^{2}\right) \Delta^{(+)}(x-i \eta, s),
\end{aligned}
$$

where

$$
\begin{aligned}
\psi_{i}\left(s, \xi^{2}\right) & =4 \pi \int_{0}^{\infty} u^{2} d u\left(\frac{\sin u \xi}{u \xi}\right) \Psi_{i}(s, u) \\
& =\int d^{3} u e^{i \mathrm{u} \cdot \xi} \Psi_{i}(s, u)
\end{aligned}
$$

and

$$
\xi_{ \pm}^{2}=(x \mp i \eta)^{2}+[p(x \mp i \eta) / m]^{2} .
$$

A Lorentz transformation has been used to obtain the commutator in an arbitrary frame from that in the rest frame. The variable $\xi^{2}$ is just the magnitude of the spatial coordinate in the rest frame.

Since the integral must exist and $\Psi_{i}(s, u)$ has finite support in $u, \psi_{i}$ may be expanded in a power series in $\xi^{2}$ :

$$
\psi_{i}\left(s, \xi^{2}\right)=\sum_{n=0}^{\infty} \xi^{2 n} \psi_{i}^{(n)}(s)
$$

where

$$
\begin{aligned}
\psi_{i}^{(n)}(s) & =(-1)^{n}[(2 n+1) !]^{-1} \int d^{3} u|\mathbf{u}|^{2 n} \Psi_{i}(s,|\mathbf{u}|) \\
& =(-1)^{n}[(2 n+1) !]^{-1} 4 \pi \int_{0}^{\infty} d u u^{2(n+1)} \Psi_{i}(s, u)
\end{aligned}
$$

This expansion is then absolutely and uniformly convergent for fixed $s$. The next problem is to discuss the asymptotic behavior of $\psi_{i}^{(n)}(s)$. In order to do this, it is useful to consider a modified Mellin transform:

$$
g_{i}{ }^{(n)}(\alpha)=\int_{0}^{\infty} d s \psi_{i}{ }^{(n)}(s)\left[1+\left(s / m^{2}\right)\right]^{\alpha-1}
$$

The transform is taken with respect to $\left[1+\left(s / m^{2}\right)\right]$ instead of $s$ in order to avoid a spurious singularity at $s=0$. Then, assuming that $\psi_{i}^{(n)}(s)$ is polynomial bounded, $g_{i}{ }^{(n)}(\alpha)$ is analytic for $\operatorname{Re} \alpha<-M$, where $\lim _{s \rightarrow \infty} s^{-M} \psi_{i}^{(n)}(s)=0$, and we assume that $M$ can be taken independent of $n$. The inverse transform is

$$
\begin{aligned}
& \psi_{i}^{(n)}(s)=(2 \pi i)^{-1} \int_{-\sigma-i \infty}^{-\sigma+i \infty} d \alpha g_{i}{ }^{(n)}(\alpha) m^{-2} \\
& \times\left[1+\left(s / m^{2}\right)\right]^{-\alpha}
\end{aligned}
$$

$$
\sigma \geq M
$$

and we assume that the asymptotic behavior of $g_{i}{ }^{(n)}(\alpha)$, as $\alpha \rightarrow \infty$, is such that this inverse exists.

The element $\left\langle p\left|j^{\mu} j^{\nu}\right| p\right\rangle$ is then given by a sum of terms of the form $\xi^{2 n} \chi^{n}(\zeta)$, where

$$
\begin{aligned}
\chi^{(n)}(\zeta)= & \int_{0}^{\infty} d s \psi_{i}{ }^{(n)}(s) \Delta(\zeta, s) \\
= & \int_{0}^{\infty} d s \psi_{i}^{(n)}(s)\left(s \zeta^{-1}\right)^{1 / 2}(2 \pi)^{-2} K_{1}\left((s \zeta)^{1 / 2}\right) \\
= & (2 \pi i)^{-1} \int_{-\sigma-i \infty}^{-\sigma+i \infty} d \alpha g_{i}^{(n)}(\alpha) m^{2(\alpha-1)} \\
& \times \int_{0}^{\infty} d s\left(m^{2}+s\right)^{-\alpha}(2 \pi)^{-2}\left(s \zeta^{-1}\right)^{1 / 2} K_{1}\left((s \zeta)^{1 / 2}\right) \\
= & (2 \pi i)^{-1} \int_{-\sigma-i \infty}^{-\sigma+i \infty} d \alpha g_{i}^{(n)}(\alpha)\left(m^{2} \zeta\right)^{\alpha / 2} \\
& \quad \times\left[(2 \pi)^{2} \zeta\right]^{-14} S_{-\alpha-1,-\alpha+2}\left(\left(m^{2} \zeta\right)^{1 / 2}\right),
\end{aligned}
$$

where $S_{\mu, \nu}(z)$ is Lommel's function of the second kind. ${ }^{19}$ The asymptotic behavior of this function for small $z$ and $\operatorname{Re} \alpha<2$ is given by

$$
\begin{aligned}
& 4 z^{\alpha} S_{-\alpha-1,-\alpha+2}(z) \equiv(\alpha-1)^{-1}\left(1-\left(\frac{1}{2} z\right)^{2} \Gamma(2-\alpha) \sum_{n=0}^{\infty}\left(-\frac{1}{4} z^{2}\right)^{m}\right. \\
& \quad \times\left[\ln \left(\frac{1}{4} z^{2}\right)-\psi(3+m-\alpha)-\psi(m+1)\right] \\
& \quad \times[m ! \Gamma(3+m-\alpha)]^{-1}-\left(\frac{1}{2} z\right)^{\alpha} \pi \Gamma(2-\alpha) \\
& \left.\quad \times\left[J_{2-\alpha}(z) \cot \pi(2-\alpha)-J_{\alpha-2}(z) \csc \pi(2-\alpha)\right]\right) \\
& \sim(\alpha-1)^{-1}\left(1+\left(\frac{1}{4} z^{2}\right)^{\alpha-1} \Gamma(2-\alpha) \sum_{m=0}^{[\operatorname{Re}(2-\alpha)]}\left(\frac{1}{4} z^{2}\right)^{m}\right. \\
& \left.\quad \times \Gamma(2-\alpha-m) m !^{-1}\right)+O\left(z^{2}\right), \quad z \rightarrow 0
\end{aligned}
$$

while, for $\operatorname{Re} \alpha \geq 2$,

$$
\begin{aligned}
& 4 z^{\alpha} S_{-\alpha-1,-\alpha-2}(z) \\
& \sim(\alpha-1)^{-1}\left[1+\frac{1}{4} z^{2}(\alpha-2)^{-1} \ln \left(\frac{1}{4} z^{2}\right)\right], \quad z \rightarrow 0
\end{aligned}
$$

where $[a] \equiv$ largest integer less than $a$.

We have dropped a term $z^{2} \ln z$ in the $\operatorname{Re} \alpha<2$ expansion; it vanishes for integer $(2-\alpha)$, which is the only case where it would be needed. The $\alpha$ integral for $g^{(n)}$ can then be performed by moving the contour to the right until $1-n<\operatorname{Re} \alpha<2-n$. The only singularities

${ }^{19}$ The integral over $s$ may be performed by referring to the Bateman Manuscript Project, edited by A. Erdelyi (McGrawHill Book Co., New York, 1954), Vol. II, p. 128 (8). The properties of Lommel's function are obtained from G. N. Watson, Theory of Bessel Functions (Cambridge University Press, London, 1958), 2nd ed., p. 348. 
are those of $g_{i}^{(n)}(\alpha)$ and the result is

$$
\begin{aligned}
& \int_{0}^{\infty} d s \psi_{i}^{(n)}(s) \Delta(\zeta, s) \underset{\zeta \rightarrow 0}{\sim}\left[(2 \pi)^{2} \zeta\right]^{-1} \\
& \times\left[(2 \pi i)^{-1} \int_{\operatorname{Re} \alpha<\frac{3}{2}-n} d \alpha \operatorname{disc} g_{i}^{(n)}(\alpha)(\alpha-1)^{-1}\right. \\
& \quad \times\left(1-\sum_{p=0}^{[\operatorname{Re}(2-\alpha)]}\left(\frac{1}{4} m^{2} \zeta\right)^{p+\alpha-1} \Gamma(2-\alpha) \Gamma(2-\alpha-p) p !^{-1}\right) \\
& +(2 \pi i)^{-1} \int_{\frac{3}{3}-n-i \infty}^{\frac{3}{2}-n+i \infty} d \alpha g_{i}^{(n)}(\alpha)(\alpha-1)^{-1} \\
& \left.\quad \times\left[1-\left(\frac{1}{4} m^{2} \zeta\right)^{\alpha-1} \Gamma^{2}(2-\alpha)\right]\right]+O\left(\zeta^{-\frac{1}{2}-n}\right) .
\end{aligned}
$$

If $g_{i}{ }^{(n)}(\alpha)$ is regular at $\alpha=1$ the first terms, for $n=0$, can be combined to form

$$
\begin{aligned}
g_{i}{ }^{(0)}(1) \equiv \frac{1}{2 \pi i}\left[\int_{\operatorname{Re} \alpha<\frac{3}{2}} d \alpha \operatorname{disc} g_{i}{ }^{(n)}(\alpha)(\alpha-1)^{-1}\right. & \\
& \left.\quad+\int_{\frac{3}{2}-i \infty}^{\frac{3}{2}+i \infty} d \alpha(\alpha-1)^{-1} g_{i}{ }^{(n)}(\alpha)\right]
\end{aligned}
$$

and the integral becomes

$$
\begin{aligned}
& \frac{\delta_{n}{ }^{0} g_{i}{ }^{(0)}(1)}{(2 \pi)^{2} \zeta}-\frac{1}{2 \pi i} \int_{\operatorname{Re} \alpha<\frac{3}{2}-n} d \alpha \frac{\operatorname{disc}_{i}{ }^{(n)}(\alpha)}{\alpha-1} \\
& \quad \times \sum_{p=0}^{[\operatorname{Re}(2-\alpha)]}\left(\frac{1}{4} m^{2} \zeta\right)^{p+\alpha-1} \frac{\Gamma(2-\alpha) \Gamma(2-\alpha-p)}{p ! \zeta(2 \pi)^{2}}
\end{aligned}
$$

for the asymptotic form as $\zeta \rightarrow 0$. The assumption of regularity at $\alpha=1$, for $n=0$, can be removed with a slight complication of the formula; however, in that case, there is either no contribution to the commutator, or the commutator does not exist, so only the case of $g^{(0)}(\alpha)$ analytic at $\alpha=1$ will be considered in this paper. The only assumption which has gone into this treatment is that the $\psi_{i}^{(n)}(s)$ are polynomial bounded.

It is now straightforward to calculate the equal-time commutators. At unequal times,

$$
\begin{aligned}
& \left\langle p\left|j^{\mu}(x) j^{\nu}(0)\right| p\right\rangle \\
& \quad=e^{i p x} \lim _{\eta \rightarrow 0}-^{-i p(x-i \eta)}\left\langle p\left|j^{\mu}(x-i \eta) j^{\nu}(0)\right| p\right\rangle
\end{aligned}
$$

and

$$
\begin{aligned}
& \left\langle p\left|j^{\nu}(0) j^{\mu}(x)\right| p\right\rangle \\
& \quad=e^{i p x} \lim _{\eta \rightarrow 0} e^{i p(x+i \eta)}\left\langle p\left|j^{\nu}(0) j^{\mu}(x+i \eta)\right| p\right\rangle ;
\end{aligned}
$$

hence,

$$
\begin{aligned}
& \left\langle p\left|\left[j^{\mu}(x), j^{\nu}(0)\right]\right| p\right\rangle \\
& \quad=\lim _{\eta \rightarrow 0} e^{p \eta}\left\langle p\left|\left(j^{\mu}(x-i \eta) j^{\nu}(0)-j^{\nu}(0) j^{\mu}(x+i \eta)\right)\right| p\right\rangle .
\end{aligned}
$$

If the commutator exists as a distribution in $\mathbf{r}$, the factor $e^{p \eta}$ can be dropped, yielding

$$
\begin{aligned}
& \left\langle p\left|\left[j^{\mu}(x), j^{\nu}(0)\right]\right| p\right\rangle \\
& =\lim _{\eta \rightarrow 0}\left\{\left(\partial^{\mu} \partial^{\nu}-g^{\mu \nu} \partial^{2}\right) \sum_{n=0}^{\infty} \int_{0}^{\infty} d s \psi_{1}{ }^{(n)}(s)\right. \\
& \quad \times\left[\xi_{+}{ }^{2 n} \Delta\left(\zeta_{+}, s\right)-\xi_{-}{ }^{2 n} \Delta\left(\zeta_{-}, s\right)\right]+\left(1 / m^{2}\right)\left[p^{\mu} p^{\nu} \partial^{2}\right. \\
& \left.-(p \partial)\left(p^{\mu} \partial^{\nu}+\partial^{\mu} p^{\nu}\right)+g^{\mu \nu}(p \partial)^{2}\right] \sum_{n=0}^{\infty} \int_{0}^{\infty} d s \psi_{2}{ }^{(n)}(s) \\
& \left.\quad \times\left[\xi_{+}{ }^{2 n} \Delta\left(\zeta_{+}, s\right)-\xi_{-}{ }^{2 n} \Delta\left(\zeta_{-}, s\right)\right]\right\},
\end{aligned}
$$

where

$$
\xi_{ \pm}^{2}=\zeta_{ \pm}+[p(x \mp i \eta) / m]^{2} \text { and } \zeta_{ \pm}=(x \mp i \eta)^{2} .
$$

For this to be a reasonable definition, the limit must be independent of the way in which $\eta^{\mu} \rightarrow 0$ and for the equal-time commutator to exist, the limit must exist, as a distribution in $\mathbf{r}$, at $t=0$ for all $\mathbf{p}$.

We first consider the time-time components of the equal-time commutators. Here, the tensor factors only contain spatial derivatives, hence, we may simply investigate the properties of the invariant functions themselves - the spatial derivative of a distribution in $\mathbf{r}$ being well defined if the original distribution is. At $t=0$ the invariant function must be odd in $\mathbf{r}$ after the change of variables which is made below, and only odd testing functions contribute. The following integral determines the equal-time commutator:

$$
\begin{aligned}
\lim _{\eta \rightarrow 0}\left(\int d^{3} r \varphi(\mathbf{r})\right. & \sum_{n} \int_{0}^{\infty} d s \psi^{(n)}(s) \\
& \left.\times\left[\xi_{+}{ }^{2 n} \Delta\left(\zeta_{+}, s\right)-\xi_{-}{ }^{2 n} \Delta\left(\zeta_{-}, s\right)\right]\right),
\end{aligned}
$$

where, here,

$$
\xi_{ \pm}^{2}=(\mathbf{r} \mp i \eta)^{2}+\left(\eta^{0}\right)^{2}+\left[\left(\mathbf{p} \cdot \mathbf{r}-i \mathbf{p} \cdot \boldsymbol{\eta}+i p^{0} \eta^{0}\right) / m\right]^{2}
$$

and

$$
\zeta_{ \pm}=(\mathbf{r} \mp i \boldsymbol{\eta})^{2}+\left(\eta^{0}\right)^{2}
$$

Now, a change of variables may be made, $\mathbf{r} \rightarrow \pm \mathbf{r}+\boldsymbol{i} \boldsymbol{\eta}$ $-i\left(\mathbf{p} / p^{0}\right) \eta^{0}$, and the integral becomes

$$
\begin{aligned}
\lim _{\eta \rightarrow 0}\left(2 \int d^{3} r \varphi\left(\mathbf{r}+i \boldsymbol{\eta}-i \mathbf{p} \eta^{0} / p^{0}\right)\right. \\
\left.\times \sum_{n} \int_{0}^{\infty} d s \psi_{i}^{(n)}(s) \xi^{2 n} \Delta\left(\bar{\xi}_{+}, s\right)\right),
\end{aligned}
$$

where

$$
\begin{aligned}
\xi^{2} & =r^{2}+(\mathbf{p} \cdot \mathbf{r} / m)^{2}, \\
\bar{\zeta}_{+} & =r^{2}+\left(m^{2} \eta^{02} / p^{02}\right)-2 i\left(\mathbf{p} \cdot \mathbf{r} \eta^{0} / p^{0}\right) .
\end{aligned}
$$

The requirement that the limit be independent of the particular limiting procedure chosen is satisfied if the 
integral

$$
\lim _{\eta \rightarrow 0}\left(2 \int d^{3} r \varphi(\mathbf{r}) \sum_{n=0} \int d s \psi_{i}^{(n)}(s) \xi^{2 n} \Delta\left(\bar{\zeta}_{+}, s\right)\right)
$$

exists for an arbitrary odd testing function.

The time-space components have a product of a single-time derivative and a spatial derivative. The space derivative can be ignored as before and the resultant distribution arises from the first time derivative of the invariant functions. At $t=0$, the functions are even under $\mathbf{r} \rightarrow-\mathbf{r}$ and

$$
\lim _{\eta \rightarrow 0}\left(2 \int d^{3} r \varphi(\mathbf{r}) \partial^{0} \sum_{n} \int_{0}^{\infty} d s \psi_{i}^{(n)}(s) \xi^{2 n} \Delta\left(\bar{\zeta}_{+}, s\right)\right)
$$

must exist for any even testing function. Similar conditions may be written for any higher number of time derivatives. These occur in the space-space commutators and the $\left[\partial^{0} j^{k}-\partial^{k} j^{0}, j^{l}\right]$ commutator. However, the former does not yield any new conditions (the latter will be discussed later). The fact that the space-space commutators are no more singular to define than the spacetime ones should not be too surprising since in both cases we are dealing with the same model-dependent set of Schwinger terms. The existence of such terms in one part of $\left[j^{\mu}(\mathbf{r}) j^{\nu}(0)\right]$ then, in general, implies their presence in any other by the Lorentz structure of the equal time terms.

The integrals are sums of terms of the form $\xi^{2 n} / \zeta^{\beta}$ and, for $\eta \rightarrow 0$, only $\mathbf{r}=0$ contributes; thus testing functions $\varphi$ polynomial in $\mathbf{r}$ (together with a damping factor for good behavior as $\mathbf{r} \rightarrow \infty$ ) suffice to calculate the commutator. The indicated choice for odd $\varphi$ is thus $\hat{p} \cdot \mathbf{r} r^{2 t} \xi^{2 q}$ and for even $\varphi, r^{2 t} \xi^{2 q}$, times an appropriate damping function. Then, for odd testing functions

$$
\begin{gathered}
2 \int d^{3} r \hat{p} \cdot \mathbf{r} r^{2 t} \xi^{2 q} \sum_{n} \int_{0}^{\infty} d s \psi_{i}^{(n)}(s) \xi^{2 n} \Delta\left(\bar{\xi}_{+}, s\right) \\
=-\sum_{n}(2 \pi i)^{-1} \int_{\operatorname{Re} \alpha<\frac{3}{2}-n} d \alpha(\alpha-1)^{-1} \operatorname{disc}_{i}^{(n)}(\alpha) \\
\quad \sum_{p=0}^{[\operatorname{Re}(2-\alpha)]}\left(\frac{4}{m^{2}}\right)^{-1 \alpha-p} \Gamma(2-\alpha) \Gamma(2-\alpha-p) \\
\times C(n+q, t, 2-\alpha-p) \eta^{2(n+q+t+p+\alpha)},
\end{gathered}
$$

where

$$
C(n, t, \beta) \equiv 2 \int \frac{d^{3} r}{(2 \pi)^{2}} \hat{p} \cdot \mathbf{r} \frac{\xi^{2 n} r^{2 t}}{\zeta^{\beta}} \eta^{2(\beta-n-t-2)}
$$

is independent of $\eta$.

The requirement that the commutators exist as a distribution implies that this function must have a limit as $\eta \rightarrow 0$. But the explicit $\eta$ dependence shows that this can be true only if all the coefficients of the $\eta^{-\bullet}$ terms vanish (except if $\alpha \geq-n-q-t-m$ ). This leads to a set of linear equations for $\operatorname{disc}_{i}{ }^{(n)}(\alpha)$, one for each pair $(q, t)$. However, $C(n, t, \beta)$ is a function of $\mathbf{p}$ which depends on $n$. It is shown in Appendix A that, if $\alpha$ is nonintegral, the function is sufficiently complicated to ensure that the only solution, for all $\mathbf{p}$, is for the $\operatorname{disc} g_{i}{ }^{(n)}(\alpha)$ to vanish. Thus $g_{i}{ }^{(n)}(\alpha)$ must be meromorphic with simple poles at the integers less than or equal to $-n$ if the commutator is to exist. Application of the same arguments to the first time derivative implies meromorphy for the $\alpha \leq-n+1$, except for $g_{i}{ }^{(0)}(\alpha)$, which must be analytic at $\alpha=1$.

The space-space components of the commutators can be shown to exist without any further restrictions, provided the other components do. To continue to the commutator of $K^{0 l}=\partial^{0} j^{l}-\partial^{l} j^{0}$ with $j^{k}$, which involves third time derivatives, requires further analytic continuation of the $g_{i}{ }^{(n)}(\alpha)$. In order that this commutator exist, $g_{i}{ }^{(n)}(\alpha)$ must be meromorphic with simple poles at the negative integers for $\operatorname{Re} \alpha \leq-n+2, g_{i}{ }^{(1)}(\alpha)$ analytic at $\alpha=1$, and $g_{i}{ }^{(0)}(\alpha)$ analytic at $\alpha=1$ and 2 .

In order to perform an explicit calculation of the equal-time commutators, it is convenient to further restrict the singularities of the $g_{i}^{(n)}(\alpha)$. A pole of $g_{i}{ }^{(n)}(\alpha)$ at $\alpha=-n-p$ implies that

$$
\psi_{i}^{(n)}(s) \underset{s \rightarrow \infty}{\sim} s^{n+p}
$$

one would expect, a priori, that this would, in turn, require $p+1$ subtractions for the $s$ integral in the JostLehmann representation to exist. This is not the case; we show in the next section that, if the only singularities of $g_{i}{ }^{(n)}$ are for $p=0$, then the existence of the equaltime commutators implies that there are cancellations between the terms with different $n$ 's such that JostLehmann representation is unsubtracted. In the more general case, where there are singularities for $p \leq P$, a similar result holds: It can be shown that there are then at most $P$ subtractions in the representation.

The calculation of the various commutators is given in Appendix B. The result is that for the single time derivative (i.e., the space-time components of the commutator) to exist, the residues $\bar{g}^{(n)}(-n)$, defined by

$$
g^{(n)}(\alpha) \sim-(\alpha+n)^{-1} \bar{g}^{(n)}(-n)
$$

must satisfy

$$
\sum \bar{g}^{(n)}(-n) m^{-2(n+1)}(2 n+1) !=0 .
$$

This one condition is actually sufficient to guarantee that all the $\left(j^{\mu}, j^{\nu}\right)$ commutators exist and that their Lorentz transformation properties are those of a tensor.

The coefficient of the $\delta$ function in

$$
\left.\partial^{0} \int_{0}^{\infty} d s \psi\left(s, \xi^{2}\right) \Delta(\zeta, s)\right|_{t=0}
$$


is then given by

$$
\begin{aligned}
\lim _{\eta \rightarrow 0}\left\{\left.\int d^{3} r \partial^{0} 2 \int_{0}^{\infty} d s \psi\left(s, \xi^{2}\right) \Delta\left(\zeta_{+}, s\right)\right|_{t=0} i\right. & {\left[\left[g^{(0)}(1)+\bar{g}^{(0)}(0)\right]+\sum_{n=1}\left[\bar{g}^{(n)}(-n+1)+n \bar{g}^{(n)}(-n)\right] n !(n-1) !\right.} \\
& \left.\left.\times\left(\frac{4}{m^{2}}\right)^{n}\left(\frac{2 \Gamma\left(n+\frac{3}{2}\right)}{n ! \Gamma\left(\frac{1}{2}\right)}+2\left[\left(p^{0} / m\right)^{2}-1\right] n \times \sum_{r=0}^{n-1}\left(p^{0} / m\right)^{2 r} a_{r}(n-1)\right)\right]\right\},
\end{aligned}
$$

where

$$
a_{r}(n)=-\sum_{s=0}^{r} \frac{\Gamma\left(n+1-s+\frac{1}{2}\right) r ! \Gamma\left(s-\frac{1}{2}\right)}{4(n+1-s) ! s ! \Gamma\left(r+\frac{1}{2}\right) \Gamma\left(\frac{3}{2}\right)}
$$

and the $\bar{g}^{(n)}(-n)$ obey the previous constraint.

The commutator $\left\langle p\left|\left[j^{0}, j^{k}\right]\right| p\right\rangle$ is then given by

$$
\begin{aligned}
& \left\langle p\left|\left[j^{0}(\mathbf{r}), j^{k}(0)\right]\right| p\right\rangle \\
& =i \partial_{l} \delta(\mathbf{r})\left\{\delta ^ { k l } \left[g_{1}{ }^{(0)}(1)+\bar{g}_{1}{ }^{(0}(0)+\sum_{n=1}\left[\bar{g}_{1}{ }^{(n)}(-n+1)+n \bar{g}_{1}{ }^{(n)}(-n)\right]\left(\frac{4}{m^{2}}\right)^{n} n !(n-1) !\left(\frac{2 \Gamma\left(n+\frac{3}{2}\right)}{n ! \Gamma\left(\frac{1}{2}\right)}+2 n(p / m)^{2}\right.\right.\right. \\
& \left.\left.\quad \times \sum_{r=0}^{n-1}\left(p^{0} / m\right)^{2 r} a_{r}(n-1)\right)\right]+\left(\delta^{k l} p^{02}-p^{k} p^{l}\right) m^{-2}\left[g_{2}{ }^{(0)}(1)+\bar{g}_{2}{ }^{(0)}(0)+\sum\left[\bar{g}_{2}{ }^{(n)}(-n+1)+n \bar{g}_{2}{ }^{(n)}(-n)\right]\right. \\
& \left.\left.\quad \times\left(\frac{4}{m^{2}}\right)^{n} n !(n-1) !\left(\frac{2 \Gamma\left(n+\frac{3}{2}\right)}{n ! \Gamma\left(\frac{1}{2}\right)}+2 n(p / m)^{2} \sum_{r=0}^{n-1}\left(p^{0} / m\right)^{2 r} a_{r}(n-1)\right)\right]\right\}+(\nabla)^{3} \delta(\mathbf{r}) \text { terms. }
\end{aligned}
$$

The quantities $\left[g_{i}{ }^{(0)}(1)+\bar{g}_{i}{ }^{(0)}(0)\right]$ and $\left[\bar{g}_{i}{ }^{(n)}(-n+1)\right.$ $\left.+n \bar{g}_{i}{ }^{(n)}(-n)\right]$ are necessary and sufficient to determine the matrix element of the commutator. Each term has a coefficient with a different tensor structure; the unsummed terms have just the kinematical structure, while the rest transform as higher-rank tensors. Thus, the commutator, in addition to having the transformation properties dictated by the tensor structures, may transform as the pure time component of a tensor of arbitrarily high rank. ${ }^{20}$ This is just a realization of the fact that the Lorentz transformation properties of two operators do not alone determine the Lorentz transformation properties of their equal-time commutator-an arbitrary number of pairs of additional time indices is always permitted. (TCP determines whether the total number of indices is even or odd.)

A simple illustration of such higher-rank structure occurred for currents bilinear in boson fields in Sec. II. Their $\left[j^{0}, j^{l}\right]$ commutator had terms of the form $\delta^{k l}, \delta^{k l} p^{02}$, and $p^{k} p^{l}$, the latter two having unrelated coefficients. In the present notation, this means the combinations $g_{1}{ }^{(0)}(1)+\bar{g}_{1}{ }^{(0)}(0), \bar{g}_{1}{ }^{(1)}(0)+\bar{g}_{1}{ }^{(1)}(-1)$, and $g_{2}{ }^{(0)}(1)+\bar{g}_{2}{ }^{(0)}(0)$ are nonvanishing, while all the others are zero. We also observe that, in general, the $\bar{g}^{(n)}(-n+1)$ are sufficient to generate any Lorentz transformation properties of the commutator; the more singular structures $\bar{g}^{(n)}(-n)$ may be taken to be zero. These, however, do determine the $\left[j^{0}, j^{0}\right]$ commutator and part of the $\left[j^{k}, j^{l}\right]$ commutator, as well as higherorder derivative terms.

We now turn to the matrix element of the $\left[K^{0 l}, j^{k}\right]$ commutator. As was mentioned above, the region of analyticity of the $g_{i}{ }^{(n)}(\alpha)$ must be extended to the right, so that the only singularity of $g^{(n)}(\alpha)$ is at $\alpha=-n+2$ and is of the form $-\bar{g}^{(n)}(-n+2) /(\alpha+n-2)$, and $g^{(0)}(2)$ and $g^{(1)}(1)$ must be finite. Then, the asymptotic form of the unordered product is

$$
\begin{gathered}
\left\langle p\left|j^{\mu}(x) j^{\nu}(0)\right| p\right\rangle \sim\left\{( \partial ^ { \mu } \partial ^ { \nu } - g ^ { \mu \nu } \partial ^ { 2 } ) \left[\frac{g_{1}{ }^{(0)}+\bar{g}_{1}{ }^{(0)}(0)}{(2 \pi)^{2} \zeta}+\sum_{n=1} \frac{\xi^{2 n}}{(2 \pi)^{2} \xi^{n+1}}\left[\bar{g}_{1}{ }^{(n)}(-n+1)+n \bar{g}_{1}{ }^{(n)}(-n)\right](n-1) ! n !\left(\frac{4}{m^{2}}\right)^{n}\right.\right. \\
-m^{2}\left[g_{1}{ }^{(0)}(2)-g_{1}{ }^{(0)}(1)-\frac{1}{2} \bar{g}_{1}{ }^{(0)}(0)\right] \frac{\ln \zeta}{4(2 \pi)^{2}}+\frac{\xi^{2}}{(2 \pi)^{2} \zeta}\left[g_{1}{ }^{(0)}(1)+\bar{g}_{1}{ }^{(1)}(0)+\frac{1}{2} \bar{g}_{1}{ }^{(1)}(-1)\right]+\sum_{n=2} \frac{\xi^{2 n}}{(2 \pi)^{2} \zeta^{n}}\left[\bar{g}_{1}{ }^{(n)}(-n+2)\right. \\
\left.\left.+(n-1) \bar{g}_{1}{ }^{(n)}(-n+1)+\frac{1}{2} n(n-1) \bar{g}_{1}(n)(-n)\right](n-1) !(n-2) !\left(\frac{4}{m^{2}}\right)^{n-1}+\sum_{n=0} \frac{\xi^{2 n} \bar{g}_{1}{ }^{(n)}(-n)}{(2 \pi)^{2} \zeta^{n+2}}\left(\frac{4}{m^{2}}\right)^{n+1}(n+1) ! n !\right] \\
\left.+\left(\frac{1}{m^{2}}\right)\left[p^{\mu} p^{\nu} \partial^{2}-p \partial\left(p^{\mu} \partial^{\nu}+\partial^{\mu} p^{\nu}\right)+g^{\mu \nu}(p \partial)^{2}\right]\left[g_{1} \rightarrow g_{2}\right]\right\}, \quad x \rightarrow 0 .
\end{gathered}
$$

${ }^{20}$ This possibility has been noted in other contexts by D. G. Boulware and S. Deser, J. Math. Phys. 8, 1468 (1967); cf. also S. Berman and Y. Frishman, Phys. Rev. 105, 1555 (1968). 
In Appendix B we find that this expression implies that

$$
\begin{array}{r}
\left\langle p\left|\left[\left(\partial^{0} j^{l}-\partial^{l} j^{0}\right)(\mathbf{r}), j^{k}(0)\right]\right| p\right\rangle=i \delta(\mathbf{r})\left\{\delta ^ { k l } \left[m^{2}\left[g_{1}{ }^{(0)}(2)-g_{1}{ }^{(0)}(1)-\frac{1}{2} \bar{g}_{1}{ }^{(0)}(0)\right]+\left[g_{1}{ }^{(1)}(1)+\bar{g}_{1}{ }^{(1)}(0)+\frac{1}{2} \bar{g}_{1}{ }^{(1)}(-1)\right]\right.\right. \\
\times\left(6+8 p^{2} / m^{2}\right)+\sum_{n=2}\left[\bar{g}_{1}^{(n)}(-n+2)+(n-1) \bar{g}_{1}{ }^{(n)}(-n+1)+\frac{1}{2} n(n-1) \bar{g}_{1}^{(n)}(-n)\right] \\
\left.\times\left(\frac{4}{m^{2}}\right)^{n-1}(n-1) !(n-2) !\left(\frac{8 \Gamma\left(n+\frac{3}{2}\right)}{(n-1) ! \Gamma\left(\frac{1}{2}\right)}+4\left(p^{2} / m^{2}\right) n \sum_{r=0}^{n-1}\left(p^{0} / m\right)^{2 r} \tilde{a}_{r}(n-1)\right)\right] \\
\left.+\left(\delta^{k l} p^{02}-p^{k} p^{l}\right)\left[g_{1} \rightarrow g_{2}\right]\right\}+\nabla^{2} \delta(\mathbf{r}) \text { terms }+\nabla^{4} \delta(\mathbf{r}) \text { terms }
\end{array}
$$

where

$\tilde{a}_{r}(n-1)=2 n(n+1) a_{r}(n-1)-(n-1)(2 n+1) a_{r}(n-2)$.

Again the commutator is an arbitrary rank tensor since the $g$ 's are all necessary and sufficient to describe it and any powers of $p^{0}$ that may occur in the sums.

We shall see in Sec. $V$ that the quadratic and logarithmic mass divergences (if they are the leading ones) are still determined by the equal time commutators of $\left[j^{0}, j^{l}\right]$ and $\left[K^{0 l}, j^{k}\right]$. Also, under the conditions we have derived for the commutators to exist, the behavior of the functions $M^{\mu \nu}$ as $k^{0} \rightarrow \infty$ is just a polynomial plus a $1 / k^{0}$ term plus terms which vanish faster than $1 / k^{0}$. In configuration space, it is only the $1 / k^{0}$ term which yields the commutator. Hence, assuming the commutator exists, we can always identify it as the coefficient of $1 / k^{0}$. The appearance of higher-rank tensors in the commutators simply implies that in the asymptotic behavior of the $M_{i}$ there are terms of the form $\nu^{2 n} / k^{2(n+1)}$ which must be included (if the $\bar{g}^{(n)}(-n)$ are not zero there are also terms of the form $\left.\left(\nu^{2} / k^{2}\right)^{n}\right)$.

\section{JOST-LEHMANN FORMEWHEN COMMUTATORS EXIST}

In this section we discuss the properties of the JostLehmann representation in the case where the commutators exist. We deal explicitly with the class of spectral functions which was discussed in the previous section. There we saw that $g_{i}{ }^{(n)}(\alpha)$ is analytic for $\operatorname{Re} \alpha<-n$ so that

$$
\begin{array}{ll}
\psi^{(n)}(s)=(2 \pi i)^{-1} \int_{-\sigma-i \infty}^{-\sigma+i \infty} d \alpha g^{(n)}(\alpha) m^{-2}\left[1+\left(s / m^{2}\right)\right]^{-\alpha}, & \\
g^{(0)}(\alpha)=-\frac{\bar{g}^{(0)}(0)}{\alpha}+\Delta g^{(0)}(\alpha), & \Delta g^{(0)}(\alpha) \text { analytic for } \operatorname{Re} \alpha \leq 2, \\
g^{(1)}(\alpha)=-\frac{\bar{g}^{(1)}(1)}{\alpha+1}-\frac{\bar{g}^{(1)}(0)}{\alpha}+\Delta g^{(1)}(\alpha), & \Delta g^{(1)}(\alpha) \text { analytic for } \operatorname{Re} \alpha \leq 1,
\end{array}
$$

and, for $n \geq 2$,

$$
g^{(n)}(\alpha)=-\frac{\bar{g}^{(n)}(-n)}{\alpha+n}-\frac{\bar{g}^{(n)}(-n+1)}{\alpha+n-1}-\frac{\bar{g}^{(n)}(-n+2)}{\alpha+n-2}+\Delta g^{(n)}(\alpha), \quad \Delta g^{(n)}(\alpha) \text { analytic for } \operatorname{Re} \alpha \leq-n+2,
$$

where the residues $\bar{g}^{(n)}(-n)$ satisfy the constraint

$$
\sum \bar{g}^{(n)}(-n) m^{-2(n+1)}(2 n+1) !=0 .
$$


Thus,

$$
\begin{gathered}
\psi^{(0)}(s)=\bar{g}^{(0)}(0) m^{-2}+\Delta \psi^{(0)}(s), \quad \int_{0}^{\infty} d s \Delta \psi^{(0)}(s)=g^{(0)}(1)+\bar{g}^{(0)}(0)=\Delta g^{(0)}(1), \\
\int_{0}^{\infty} d s s \Delta \psi^{(0)}(s)=\left[g^{(0)}(2)-g^{(0)}(1)-\frac{1}{2} \bar{g}^{(0)}(0)\right] m^{2}, \\
\psi^{(1)}(s)=\bar{g}^{(1)}(-1) s m^{-4}+\left[\bar{g}^{(1)}(0)+\bar{g}^{(0)}(0)\right] m^{-2}+\Delta \psi^{(1)}(s), \quad \int_{0}^{\infty} d s \Delta \psi^{(1)}(s)=g^{(1)}(1)+\bar{g}^{(1)}(0)+\frac{1}{2} \bar{g}^{(1)}(-1),
\end{gathered}
$$

and, for $n \geq 2$,

$\psi^{(n)}(s)=\bar{g}^{(n)}(-n) s^{n} m^{-2(n+1)}+\left[\bar{g}^{(n)}(-n+1)+n \bar{g}^{(n)}(-n)\right] s^{n-1} m^{-2 n}$

where

$$
+\left[\bar{g}^{(n)}(-n+2)+(n-1) \bar{g}^{(n)}(-n+1)+\frac{1}{2} n(n-1) \bar{g}^{(n)}(-n)\right] s^{n-2} m^{-2(n-1)}+\Delta \psi^{(n)}(s),
$$

$$
\lim _{s \rightarrow \infty} s^{-n+2} \Delta \psi^{(n)}(s)=0 \text {. }
$$

It is now easy to show that the Jost-Lehmann representation for the time-ordered product needs no subtractions. We divide the $s$ integral into two regions:

$$
\int_{0}^{\infty} d s d^{3} u \frac{\Psi(s,|\mathbf{u}|)}{s+(\mathbf{u}-\mathbf{k})^{2}-k^{02}-i \epsilon}=\left[\int_{0}^{s_{0}}+\int_{s_{0}}^{\infty}\right] d s \int d^{3} u \frac{\Psi(s,|\mathbf{u}|)}{s+(\mathbf{u}-\mathbf{k})^{2}-k^{02}-i \epsilon}
$$

where we take $k^{2}+s_{0} \gg 2 m|\mathbf{k}|$, and $|\mathbf{u}|$ is restricted, by the Jost-Lehmann form, to the region $\leq m$. The integral up to $s_{0}$ converges in any case; the second may not, as $s \rightarrow \infty$. However, it may be expanded:

$$
\begin{gathered}
\int_{s_{0}}^{\infty} d s d^{3} u \frac{\Psi(s,|\mathbf{u}|)}{s+(\mathbf{u}-\mathbf{k})^{2}-k^{02}-i \epsilon} \sim 4 \pi \int^{\infty} d s u^{2} d u \frac{\Psi_{i}(s, u)}{4 u|\mathbf{k}|} \ln \frac{s+(|\mathbf{k}|+u)^{2}-\left(k^{0}\right)^{2}-i \epsilon}{s+(|\mathbf{k}|-u)^{2}-\left(k^{0}\right)^{2}-i \epsilon} \\
=\int^{\infty} d s \sum_{n=0} \frac{\left(4|\mathbf{k}|^{2}\right)^{n}}{2 n+1} 4 \pi \int_{0}^{\infty} d u \frac{u^{2(n+1)} \Psi_{i}(s, u)}{\left(s+u^{2}+k^{2}\right)^{2 n+1}}=\int^{\infty} d s \sum_{n, p} \frac{\left(4|\mathbf{k}|^{2}\right)^{n}}{(2 n+1) !} \frac{(2 n+p) !}{p !}(-1)^{p} 4 \pi \int_{0}^{\infty} d u \frac{u^{2(n+p+1)} \Psi_{i}(s, u)}{\left(s+k^{2}\right)^{2 n+p+1}} \\
=\int^{\infty} d s \sum_{n, r} \frac{\left(-4|\mathbf{k}|^{2}\right)^{n}(r+n) !(2 r+1) ! \Psi^{(r)}(s)}{(2 n+1) !(r-n) !\left(s+k^{2}\right)^{r+n+1}}
\end{gathered}
$$

This $s$ integral converges, except possibly for the $n=0$ term, whose asymptotic behavior is

$$
\int^{\infty} d s \sum_{r=0} \bar{g}^{(r)}(-r) m^{-2}\left(s / m^{2}\right)^{r}\left(s+k^{2}\right)^{-r-1}(2 r+1) ! \equiv \int^{\infty} d s \sum_{r=0} \bar{g}^{(r)}(-r)(2 r+1) ! m^{-2(r+1)}\left\{\left[s /\left(s+k^{2}\right)\right]^{r}-1\right\},
$$

which is then also convergent by virtue of the constraint represented by the -1 term. Note that the absence of the condition on the $\bar{g}^{(r)}(-r)$ would lead to a subtraction and to asymptotic behavior of the form $\sim \ln k^{2}$.

Hence, we conclude that the existence of the commutator implies that the Jost-Lehmann form is, in fact, unsubtracted. If the $\psi^{(n)}(s)$ grow faster than $s^{n}$, but no faster than $s^{n+p}$ the existence of the commutator implies that the retarded commutator obeys a Jost-Lehmann representation with $p$ subtractions. 
The next problem is to relate the divergence of the mass shift to the equal-time commutator. Following a procedure similar to the earlier one, we have

$$
\begin{aligned}
& \delta m^{2}=-\left(\frac{3 \alpha}{8 \pi}\right) \int^{\infty} k^{2} d k^{2} \int d s d^{3} u\left[\frac{\Psi_{1}(s, u) 2}{s+u^{2}+k^{2}+\gamma}+\frac{1}{3}\left(1+\frac{1 s+u^{2}+k^{2}+2 \gamma}{3 s+u^{2}+k^{2}+\gamma}\right) \frac{\Psi_{2}(s, u) 2}{s+u^{2}+k^{2}+\gamma}\right] \sim-\left(\frac{3 \alpha}{8 \pi}\right) \int^{\infty} k^{2} d k^{2} \\
& \times\left(\sum_{r=0}^{\infty}(2 r+1) ! \sum_{p=0}^{r} \frac{(r+p) !\left(-k^{2}\right)^{p} \Psi_{1}^{(r)}(s)}{p !(p+1) !(r-p) !\left(s+k^{2}\right)^{r+p+1}}+\frac{1}{3} \sum_{r=0}^{\infty}(2 r+1) ! \sum_{p=0}^{r} \frac{(p+3)(r+p) !\left(-k^{2}\right)^{p} \psi_{2}{ }^{(r)}(s)}{p !(p+2) !(r-p) !\left(s+k^{2}\right)^{r+p+1}}\right) \\
& \sim-\left(\frac{3 \alpha}{8 \pi}\right) \int^{\infty} k^{2} d k^{2}\left\{-\sum_{r=1}(2 r+1) !\left[\frac{\bar{g}_{1}^{(r)}(-r)}{m^{2(r+1)}} \sum_{p=1}^{r}\left(\frac{1}{p}+\frac{1}{p+1}\right)+\frac{\bar{g}_{2}^{(r)}(-r)}{m^{2(r+1)}} \sum_{p=1}\left(\frac{1}{2 p}+\frac{1}{3(p+1)}+\frac{1}{6(p+2)}\right)\right]\right. \\
& +\left(g_{1}{ }^{(0)}(1)+\bar{g}_{1}{ }^{(0)}(0)+\sum_{r=1} \frac{(2 r+1) !}{m^{2 r}} \frac{\left[\bar{g}_{1}{ }^{(r)}(-r+1)+r \bar{g}_{1}{ }^{(r)}(-r)\right]}{(r+1) r}+\frac{1}{2}\left[g_{2}{ }^{(0)}(1)+\bar{g}_{2}{ }^{(0)}(0)\right]\right. \\
& \left.+\sum_{r=1} \frac{(2 r+1) !}{m^{2 r}} \frac{(2 r+3)\left[\bar{g}_{2}{ }^{(r)}(-r+1)+r \bar{g}_{2}{ }^{(r)}(-r)\right]}{3(r+1)(r+2)}\right) \frac{1}{k^{2}}+\left(\left(-m^{2}\right)\left[g_{1}{ }^{(0)}(2)-g_{1}{ }^{(0)}(1)-\frac{1}{2} \bar{g}_{1}{ }^{(0)}(0)\right]\right. \\
& +\frac{1}{2}\left(-m^{2}\right)\left[g_{2}{ }^{(0)}(2)-g_{2}{ }^{(0)}(1)-\frac{1}{2} \bar{g}_{2}{ }^{(0)}(0)\right]+\frac{1}{3} \times \frac{1}{6}\left[g_{2}{ }^{(1)}(1)+\bar{g}_{2}{ }^{(1)}(0)+\frac{1}{2} \bar{g}_{2}{ }^{(1)}(-1)\right] \\
& \left.\left.+\frac{1}{3} \sum_{r=2} \frac{(2 r+1) !(r-2) !}{(r+2) ! m^{2(r-1)}}\left[\bar{g}_{2}{ }^{(r)}(-r+2)+(r-1) \bar{g}_{2}{ }^{(r)}(-r+1)+\frac{1}{2} r(r-1) \bar{g}_{2}{ }^{(r)}(-r)\right]\right)\left(\frac{1}{k^{4}}\right)\right\} .
\end{aligned}
$$

While the $1 / k^{2}$ and $1 / k^{4}$ terms are indeed determined by the equal-time commutators, there is also a constant term, which ostensibly implies a quartic divergence, unrelated to the above commutators. ${ }^{21}$ It prevents the very introduction of a Wick rotation (which can handle quadratics) since in the original Lorentz metric it is nonzero as $k^{0} \rightarrow \infty$, and so the usual surface terms in the rotation would not vanish and are, in fact, complex. Thus, in order to discuss the divergences meaning- fully in the mass shift, we must deal with systems for which this contribution is absent in the original Lorentz space form to start with. Different methods of evaluating the various terms in fact yield different quartic divergences; hence, we assume that the $\bar{g}^{(n)}(-n)$ are all zero in order to eliminate unambiguously the quartic divergences. Then $g^{(n)}(\alpha)$ is analytic for $\operatorname{Re} \alpha<-n+1$.

Under these circumstances, only quadratic and logarithmic divergences occur:

$$
\begin{aligned}
& \delta m^{2} \sim-\left(\frac{3 \alpha}{8 \pi}\right) \int^{\infty} d k^{2}\left(g_{1}{ }^{(0)}(1)+\frac{1}{2} g_{2}{ }^{(0)}(1)+\sum_{r=1} \frac{(2 r+1) !}{m^{2 r}} \frac{\left[(r+2) \bar{g}_{1}{ }^{(r)}(-r+1)+\frac{1}{3}(2 r+3) r \bar{g}_{2}(r)(-r+1)\right]}{r(r+1)(r+2)}\right) \\
& -\left(\frac{3 \alpha}{8 \pi}\right) \int^{\infty} \frac{d k^{2}}{k^{2}}\left(g_{2}{ }^{(0)}(2)-g_{1}{ }^{(0)}(1)+\frac{1}{2}\left[g_{2}{ }^{(0)}(2)-g_{2}{ }^{(0)}(1)\right]+\frac{\frac{1}{3} \times \frac{1}{2}}{\left(-m^{2}\right)}\left[g_{2}{ }^{(1)}(1)+\bar{g}_{2}{ }^{(1)}(0)\right]\right. \\
& \left.-\frac{1}{3} \sum_{r=2} \frac{(2 r+1) !(r-2) !}{m^{2 r}(r+2) !}\left[g_{2}^{(r)}(-r+2)+(r-1) \tilde{g}_{2}{ }^{(r)}(-r+1)\right]\right) \text {. }
\end{aligned}
$$

We recall that the $g^{(0)}$ terms and the $g^{(1)}$ term come from the kinematic tensor structure for the commutator $C^{\mu \nu \lambda \sigma}$, while the summation comes from the possible

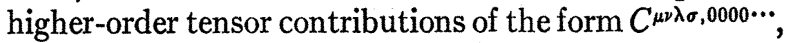
as may be seen by comparison with the previous section. It may therefore be concluded that both the quadratic and logarithmic terms in $\delta m^{2}$ are determined, in general, by the $\bar{g}_{i}(r)$ coefficients specifying the equal-

\footnotetext{
${ }^{21}$ Its presence merely means that the present framework can accommodate sufficiently singular theories to produce this high a divergence and such terms cannot be disposed of by requiring that $\left[j^{0}, j^{k}\right]$ exist. The quartic divergence is related to the $\left[j^{0}, j^{0}\right]$ commutator's $\nabla \nabla \delta(\mathbf{r})$ terms or to the third derivative terms in the $\left[j^{0}, j^{k}\right]$ commutator, i.e., to "nonleading" Schwinger terms.
}

time commutator expansions whenever these equaltime commutators exist. ${ }^{22}$

${ }^{22}$ The existence of nonvanishing equal-time commutators implies that $M_{i}$ behaves as $k^{-2}$ or $k^{-4}$. However, it is of course possible to have behavior [e.g., $\left(k^{4} \ln k^{2}\right)^{-1}$ ] such that the commutators vanish but there is a divergent mass shift (here $\delta m^{2} \sim \ln \ln \Lambda^{2}$ ). This type of behavior arises from branch points in the $g^{(n)}(\alpha)$ whose discontinuity at $\alpha=-n+2$ is less singular than $(\alpha+n-2)$ which yields $\delta m^{2}$ less divergent than $\ln \Lambda^{2}$. If this discontinuity were more singular, the commutator would not exist and $\delta m^{2}$ would be more than logarithmically divergent. In our discussion of the commutator [see text following Eq. (4.11)], we have omitted such possibilities since they would not contribute there, and would give the less singular $\delta m^{2}$ contributions illustrated above. $\mathbf{H}$. Epstein and R. Jackiw (unpublished) discuss such possibilities; we thank them for bringing these cases to our attention. 
These results can be expressed in a much more transparent form. The requirement that the equaltime commutators exist restricts the asymptotic behavior of the invariant amplitudes to sums of terms of the form $\left(1 / k^{2}\right)^{p}\left(\nu^{2} / k^{2}\right)^{r}$ for $m \nu \ll<k^{2}$ as $k^{2}$ goes to infinity. To see this, we recall that

$$
\begin{aligned}
M_{i}\left(k^{2}, \nu\right) & =\int d^{3} u d s \frac{\Psi_{i}(s,|\mathbf{u}|)}{s+(\mathbf{u}-\mathbf{k})^{2}-k^{02}-i \epsilon} \\
& =\sum_{r} \int d^{3} u d s \frac{\left(4|\mathbf{k}|^{2}\right)^{r}}{2 r+1} \frac{u^{2 r} \Psi_{i}(s, u)}{\left(s+u^{2}+k^{2}\right)^{2 r+1}} \\
& =\sum_{r, p} \frac{\left[-4\left(k^{2}+\nu^{2}\right)\right]^{r}(p+r) !(2 p+1) !}{(2 r+1) !(p-r) !} \\
& \times \int_{0}^{\infty} d s \frac{\Psi^{(p)}(s)}{\left(s+k^{2}\right)^{p+r+1}},
\end{aligned}
$$

and the leading terms of the $s$ integral are a polynomial in $1 / k^{2}$. Thus, expanding the factor $\left(k^{2}+\nu^{2}\right)$, we obtain

$$
\begin{aligned}
M_{i}\left(k^{2}, \nu\right) \underset{k^{2} \rightarrow \infty, k^{2} \gg \nu m}{\sim} & {\left[\frac{1}{k^{2}} \sum_{r=0} C_{i}\left(\frac{-\nu^{2}}{k^{2}}\right)^{r}\right.} \\
& \left.+\frac{1}{k^{4}} \sum_{r=0} \bar{C}_{i^{r}}\left(\frac{-\nu^{2}}{k^{2}}\right)^{r}\right]+O\left(\left(1 l k^{2}\right)^{2+\epsilon}\right) .
\end{aligned}
$$

If there are higher-order derivative terms in the commutators $\left[\bar{g}^{(n)}(-n) \neq 0\right]$ there will be terms of order $1\left[O\left(\left(\nu^{2} / k^{2}\right)^{r}\right)\right]$ or higher and the problems of a quartic or higher divergence in the mass shift will appear. The $C_{i}$ are linear combinations of the $\bar{g}^{(n)}(-n+1)$, hence they determine the $\left[j^{0}, j^{k}\right]$ commutator. The $\bar{C}_{i}$ are linear combinations of the

$$
\left[\bar{g}^{(n)}(-n+2)+(n-1) \bar{g}^{(n)}(-n+1)\right],
$$

hence they determine the $\left[K^{0 l}, j^{k}\right]$ commutator. This is readily seen by taking the limit as $k^{0} \rightarrow \infty, \mathbf{k}$ constant. Then

$M_{i}\left(k^{2}, \nu\right) \sim-\left(\frac{1}{k^{0}}\right)^{2} \sum_{r=0} C_{i}{ }^{r}\left(\frac{p^{02}}{m^{2}}\right)^{r}+\left(\frac{1}{k^{0}}\right)^{4} \sum_{r} \bar{C}_{i^{r}}\left(\frac{p^{02}}{m^{2}}\right)^{r}$

and the equal-time commutators are

and

$$
\left\langle p\left|\left[j^{0}(\mathbf{r}), j^{l}(0)\right]\right| p\right\rangle=i \partial_{k} \delta(\mathbf{r})\left[\delta^{k l} \sum_{r=0} C_{1^{r}}\left(\frac{p^{0}}{m}\right)^{2 r}+\frac{1}{m^{2}}\left(\delta^{k l} p^{02}-p^{k} p^{l}\right) \sum_{r=0} C_{2^{r}}\left(\frac{p^{0}}{m}\right)^{2 r}\right]
$$

$$
\left\langle p\left|\left[K^{0 l}(\mathbf{r}), j^{k}(0)\right]\right| p\right\rangle=i \delta(\mathbf{r})\left[\delta^{k l} \sum_{r=0} \bar{C}_{1^{r}}\left(\frac{p^{0}}{m}\right)^{2 r}+\frac{1}{m^{2}}\left(p^{02} \delta^{k l}-p^{k} p^{l}\right) \sum_{r} \bar{C}_{2}{ }^{r}\left(\frac{p^{0}}{m}\right)^{2 r}\right] .
$$

On the other hand, the mass shift can be calculated directly:

$$
\begin{aligned}
& \delta m^{2}=-\frac{3 \alpha}{4 \pi} \int \frac{d^{4} k}{2 \pi^{2}}\left\{M_{1}\left(k^{2}, i k_{4}\right)+\frac{1}{3}\left(1+\frac{2 k_{4}^{2}}{k^{2}}\right) M_{2}\left(k^{2}, i k_{4}\right)\right\} \\
& =-\frac{3 \alpha}{4 \pi} \int \frac{d^{4} k}{2 \pi^{2}}\left\{\frac{1}{k^{2}} \sum_{r=0} C_{1^{r}}\left(\frac{k_{4}{ }^{2}}{k^{2}}\right)^{r}+\frac{1}{3} \frac{1}{k^{2}}\left(1+\frac{2 k_{4}{ }^{2}}{k^{2}}\right) \sum_{r=0} C_{2}{ }^{r}\left(\frac{k_{4}{ }^{2}}{k^{2}}\right)^{r}\right\} \\
& \left\{+\frac{1}{k^{4}} \sum_{r=0} \bar{C}_{1^{r}}\left(\frac{k_{4}^{2}}{k^{2}}\right)^{r}+\frac{1}{3} \frac{1}{k^{4}}\left(1+\frac{2 k_{4}^{2}}{k^{2}}\right) \sum_{r=0} \bar{C}_{2^{r}}\left(\frac{k_{4}^{2}}{k^{2}}\right)^{r}\right\}+\text { finite terms } \\
& =-\frac{3 \alpha}{8 \pi} \int d k^{2}\left[\left(\sum_{r=0} C_{1^{r}} \frac{(2 r) !}{r !(r+1) !} \frac{1}{4^{r}}+\sum_{r=0} C_{2} \frac{(2 r) !(r+1)}{r !(r+2) ! 4^{r}}\right)\right. \\
& \left.+\frac{1}{k^{2}}\left(\sum_{r=0} \bar{C}_{1} \frac{(2 r) !}{r !(r+1) ! 4^{r}}+\sum_{r=0} \bar{C}_{2} \frac{(2 r) !(r+1)}{r !(r+2) ! 4^{r}}\right)\right]+ \text { finite terms. }
\end{aligned}
$$

In conclusion, we have seen that when the equal-time commutators exist they restrict the asymptotic behavior of the invariant amplitudes and determine the mass-shift divergences; asymptotic $\nu$ behavior is not relevant to the latter.

\section{ACKNOWLEDGMENTS}

We wish to thank the 1967 Summer Institute of the Niels Bohr Institute for hospitality while this work was begun. One of us (D.G.B.) also thanks Brookhaven National Laboratory and Professor Abdus Salam and the International Atomic Energy Association for hospitality at the International Centre for Theoretical Physics, Trieste, while the work was completed. 


\section{APPENDIX"A}

The coefficients which appear in Eq. (4.16) are $\left[\gamma=\left[1+(p x / m)^{2}\right]^{1 / 2}\right]$

$$
\begin{aligned}
& C(n, t, \beta) \equiv 2(2 \pi)^{-2} \int d^{3} r \hat{p} \cdot \mathbf{r} \xi^{2 n} r^{2 t} \zeta_{+}{ }^{-\beta} \eta^{2(\beta-n-t-2)} \\
& =\int_{-1}^{1} d x \int_{-\infty}^{\infty} d r(2 \pi)^{-1} r^{2(t+n)+3} x \gamma^{2 n}\left[r^{2}+\eta^{2}-2 i p r x \eta m^{-1}\right]^{-\beta} \eta^{2(\beta-n-t-2)} \\
& =\int_{-1}^{1} d x x \int_{-\infty}^{\infty} d r(2 \pi)^{-1}\left(r+i p x m^{-1}\right)^{2(t+n)+3} \gamma^{2 n}\left[r^{2}+\gamma^{2}\right]^{-\beta} \\
& =\int_{-1}^{1} d x x \gamma^{2(n-\beta)+1} \int_{-\infty}^{\infty} d r(2 \pi)^{-1}\left(r \gamma+i p x m^{-1}\right)^{2(t+n)+3}\left[r^{2}+1\right]^{-\beta} \\
& =\int_{-1}^{1} d x x \gamma^{2(n-\beta)+1} \int_{-\pi / 2}^{\pi / 2} d \theta(2 \pi)^{-1} \cos ^{2(\beta-n-t)-5} \theta\left[\gamma \sin \theta+i p x m^{-1} \cos \theta\right]^{2(t+m)+3} \\
& =i p(2 \pi m)^{-1} \int_{-1}^{1} d x x^{2} \gamma^{2(n-\beta)+1} \sum_{s} \frac{[2(t+n+1)+1] ! \gamma^{2(t+n+1-s)+1}\left(1-\gamma^{2}\right)^{s}}{(2 s+1) ![2(t+n+1-s)] !} \\
& \times \frac{\Gamma\left(t+n+1-s+\frac{1}{2}\right) \Gamma\left(\beta+s-n-t-2-\frac{1}{2}\right)}{\Gamma(\beta)} \\
& =i p(2 m)^{-1} \int_{-1}^{1} d x x^{2} \sum_{s}[2(t+n+1)+1] ! \gamma^{2(t+2 n+1-\beta-s)+1}\left(1-\gamma^{2}\right)^{s} \\
& \times \frac{\Gamma\left(\beta+s-n-t-2+\frac{1}{2}\right)}{(2 s+1) ! \Gamma(\beta) \Gamma\left(\frac{1}{2}\right)(t+n+1-s) ! 4^{t+n+1-8}} .
\end{aligned}
$$

First consider the case when $\beta$ is not an integer; we choose $t=0 ; q=0$, and $\beta>n+2$. Then let $\beta=n+2+\epsilon$ and we must sum over $n$ for fixed $\epsilon$ in order to find the coefficient of $\eta^{-2 \epsilon}$ in Eq. (4.16). Now

$$
C(n, 0, n+2+\epsilon)=i(p / 2 m) \int_{-1}^{1} d x x^{2} \gamma^{-3-2 \epsilon} \sum_{s} \gamma^{2(n+1-s)}\left(1-\gamma^{2}\right)^{s}\left(\frac{(2 n+3) ! \Gamma\left(\epsilon+s+\frac{1}{2}\right)}{(2 s+1) ! \Gamma(n+2+\epsilon) \Gamma\left(\frac{1}{2}\right)(n+1-s) ! 4^{n+1-s}}\right),
$$

which is then a sum of terms of the form $(0 \leq r \leq n+1)$

$$
\begin{aligned}
\frac{1}{2} \int_{-1}^{1} d x x^{2} \gamma^{2(r-\epsilon)-3}= & \frac{1}{2} \frac{\partial}{\partial\left(p^{2} / m^{2}\right)} \int_{-1}^{1} d x \gamma^{2\left(r-\epsilon-\frac{1}{2}\right)}\left(r-\epsilon-\frac{1}{2}\right)^{-1}=2[2(r-\epsilon)-1]^{-1} \frac{\partial}{\partial\left(p^{2} / m^{2}\right)}\left\{\left(\frac{p^{0}}{m}\right)^{2(r-\epsilon)-1}\right. \\
=2[2(r-\epsilon)- & 1]^{-1} \frac{\partial}{\partial\left(p^{2} / m^{2}\right)}\left\{(r-\epsilon)^{-1}\left(\frac{p^{0}}{m}\right)^{2\left(r-\epsilon-\frac{1}{2}\right)}+\left[\left(r-\epsilon-\frac{1}{2}\right) /(r-\epsilon)\right] \int_{-1}^{1} d x \gamma^{2(r-\epsilon-1)} d x\left(\gamma^{2}-1\right) \gamma^{2\left(r-\epsilon-\frac{1}{2}\right)}\right\} \\
= & \frac{1}{2} \frac{\partial}{\partial\left(p^{2} / m^{2}\right)}\left[\sum_{m=0}^{r-1} \frac{\Gamma(r-m-\epsilon) \Gamma\left(r-\epsilon-\frac{1}{2}\right)}{\Gamma(r+1-\epsilon) \Gamma\left(r-m-\epsilon+\frac{1}{2}\right)}\left(\frac{p^{0}}{m}\right)^{2\left(r-m-\epsilon-\frac{1}{2}\right)}+\frac{\Gamma(1-\epsilon) \Gamma\left(r-\epsilon-\frac{1}{2}\right)}{\Gamma(r+1-\epsilon) \Gamma\left(\frac{1}{2}-\epsilon\right)} \int_{-1}^{1} d x \gamma^{-1-2 \epsilon}\right] \\
= & \frac{1}{2}\left[\sum_{s=0}^{r-1} \frac{\Gamma(r-s-\epsilon) \Gamma\left(r-\epsilon-\frac{1}{2}\right)}{\Gamma(r+1-\epsilon) \Gamma\left(r-s-\epsilon-\frac{1}{2}\right)}\left(\frac{p^{0}}{m}\right)^{2\left(r-s-\epsilon-\frac{3}{2}\right)}+\frac{\Gamma(1-\epsilon) \Gamma\left(r-\epsilon-\frac{1}{2}\right)}{\Gamma(r+1-\epsilon) \Gamma\left(-\frac{1}{2}-\epsilon\right)} \int_{-1}^{1} d x x^{2} \gamma^{-2 \epsilon-3}\right] . \quad \text { (A3) }
\end{aligned}
$$

If $\epsilon$ is neither integer nor $\frac{1}{2}$-odd integer, then the series does not terminate and, since $r$ can be as large as $n+1$, we have a power series in $\left(p^{0}\right)^{2}$, multiplying $\left(p^{0} / m\right)^{-2 \epsilon^{-3}}$. The highest-order term in the series is $n+1$, hence each term in the series multiplies a linearly independent function of $p^{0}$. For the function to vanish in all Lorentz frames, each term in the series must vanish. Thus, $\operatorname{disc} g^{(n)}(\alpha)=0$, except for $\alpha$ integral or $\frac{1}{2}$-odd integral. 
We now turn to $\frac{1}{2}$-odd integral $\epsilon$; then, we choose $t=0$ and $q$ such that $\beta-n-q-2=\epsilon=\frac{1}{2}$. The same calculation then applies, except that the series terminates; the last term vanishes since $\Gamma\left(-\epsilon-\frac{1}{2}\right)=\infty$ for $\epsilon=\frac{1}{2}$. Again, the coefficients are linearly independent functions of $p^{0}$ for each $n$. This leaves the points where $\epsilon$ is integral. The argument can not be carried through now-the series terminate but the functions are not linearly independent, leaving an insufficient number of equations to determine all the $\left[\operatorname{disc} \bar{g}^{(n)}(\alpha)\right]$ at $\alpha$ integral. However, the singularity can be restricted to be a simple pole, since any multiple pole would yield a singular behavior as $\eta$ goes to zero and the coefficient of the order $n$ term is a polynomial of order $n$ in $p^{02}$. We thus conclude that the only possible behavior for $g^{n}(\alpha)$ for $\operatorname{Re} \alpha \leq-n+1$ is that $g^{(n)}(\alpha)$ is meromorphic with simple poles at the integers, i.e., $\psi^{(n)}(s)$ $\sim \sum_{m} s^{m} \bar{g}^{(n)}(m)+\tilde{\psi}$, where $s^{-n+1} \tilde{\psi} \rightarrow 0$ as $s \rightarrow \infty$. If we consider the $\left[K^{0 l}, j^{k}\right]$ commutator as well, the region about which statements may be made is moved to the right and $g^{(n)}(\alpha)$ is meromorphic for $\operatorname{Re} \alpha \leq-n+2$.

In the case where $\epsilon$ is an integer, the $C$ 's are more easily calculated using a slightly different technique $(b \equiv i p x / m)$ :

$$
\begin{aligned}
C(n, t, n+t+2+\epsilon) \\
\quad=\int_{-1}^{1} d x x \gamma^{-2(t+\epsilon)-3} \int_{-\pi / 2}^{\pi / 2} d \theta(2 \pi)^{-1} \cos ^{2 \epsilon-1} \theta[\gamma \sin \theta+b \cos \theta]^{2(t+n)+3} \\
=\int_{-1}^{1} d x x \gamma^{-2(t+\epsilon)-3}\left(\frac{\partial}{\partial b}\right)^{2 \epsilon-1} \frac{[2(t+n)+3] !}{[2(t+n+\epsilon+1)] !} \int_{-\pi / 2}^{\pi / 2} d \theta(2 \pi)^{-1}[\gamma \sin \theta+b \cos \theta]^{2(t+n+\epsilon+1)} \\
=\int_{-1}^{1} d x x \gamma^{-2(t+\epsilon)-3}\left(\frac{\partial}{\partial b}\right)^{2 \epsilon-1}\left(\gamma^{2}+b^{2}\right)^{t+n+\epsilon+1} \frac{[2(t+n+1)+1] ! \Gamma\left(t+n+\epsilon+\frac{3}{2}\right)}{2 \Gamma\left(\frac{1}{2}\right)[2(t+n+\epsilon+1)] ![t+n+\epsilon+1] !} \\
=\frac{i p}{m} \int_{-1}^{1} d x x^{2} \gamma^{-2(t+\epsilon)-3}\left[\sum_{\rho}\left(\frac{2 i p x}{m}\right)^{2 \rho} \frac{[2(t+n+1)+1] !(2 \epsilon-1) !}{2 \times 4^{t+n+\epsilon+1}(t+n+\epsilon+1) !(t+n+1-\rho) !(2 \rho+1) !(\epsilon-\rho-1) !}\right] .
\end{aligned}
$$

For fixed $t$, the result is a polynomial in $p^{2}$ whose order is independent of $n$. But $t$ can only range over a finite number of values, as can $q$; hence there are a finite number of conditions; and by using a sufficiently large number of terms we can always satisfy the conditions that the coefficients of $\eta^{-2 \epsilon}$ vanish. Similar arguments must be applied to the quantities which appear in the first time derivative; they do not change the essential result. The $g^{(n)}(\alpha)$, for $\operatorname{Re} \alpha \leq-n+1$, must be meromorphic with simple poles at the integers except that $g^{(n)}(\alpha)$ must be analytic at $\alpha=1$. The residues at the poles for $\operatorname{Re} \alpha \leq-n$ must obey a set of homogeneous linear equations; but there are no conditions on the residues at $\alpha=-n+1$.

We also need conditions for the finiteness of the commutators $\left[K^{\mu \nu}, j^{\lambda}\right]$. At least when the $g^{(n)}(\alpha)$ are analytic for $\operatorname{Re} \alpha<-n$ (as will be assumed in the text), there are no further conditions on the residues at $\alpha=-n$. However, the $g^{(n)}(\alpha)$ must be meromorphic for Re $\alpha \leq-n+2$, except that $g^{(0)}(\alpha)$ must be analytic at $\alpha=1$ and 2 and $\bar{g}^{(1)}(\alpha)$ must be analytic at $\alpha=1$.

For $\operatorname{Re} \alpha=-n+2$, the argument is more delicate since the behavior is then $\eta^{i \mathrm{Im} \alpha}$. Such functions do not possess a well-defined limit as $\eta \rightarrow 0$; we would thus regard the commutators as nonexistent if such terms occurred. Hence no singularities in $g^{(n)}(\alpha)$ are allowed away from the real axis (a branch point at $\operatorname{Re} \alpha=-n+2$ would be allowed ${ }^{22}$ provided its discontinuity were less singular than $\alpha+n-2)$. We have not considered the quite separate possibility of an isolated essential singularity.

\section{APPENDIX B}

In order to calculate the various commutators arising from the invariant functions of Sec. IV, we must evaluate several integrals. First we consider the contributions which come from the poles of $g^{(n)}(\alpha)$ at $\alpha=-n$. The relevant small-distance behavior is then $\xi^{2 n} / \zeta_{ \pm}{ }^{n+2}$. We need to calculate the properties at $t=0$ for the function and its first three time derivatives. For the function, only its odd part is relevant and we have

$$
\lim _{\eta \rightarrow 0}(2 \pi)^{-2} \xi^{2 n}\left(\zeta_{+}^{-n-2}-\zeta_{-}^{-n-2}\right)=-\hat{p} \cdot \nabla \delta(\mathbf{r}) \lambda_{n}
$$

as the only distribution that can arise is a gradient of a $\delta$ function. Here

$$
\xi^{2}=r^{2} \gamma^{2}, \quad \gamma=\left[1+(p x / m)^{2}\right]^{1 / 2}, \quad \zeta_{ \pm}=r^{2}+\eta^{2} \mp 2 i(\mathbf{p} \cdot \mathbf{r} / m) \eta
$$


and

$$
\begin{aligned}
\lambda_{n} & =\lim _{\eta \rightarrow 0} 2 \int d^{3} r(2 \pi)^{-2} \hat{p} \cdot \mathbf{r} \xi^{2 n} \zeta_{+}{ }^{-n-2} \\
& =(2 \pi)^{-1} \int_{-1}^{1} d x x \gamma^{-3} \int_{-\pi / 2}^{\pi / 2} d \theta(\cos \theta)^{-1}[\gamma \sin \theta+i(p x / m) \cos \theta]^{2 n+3} \\
& =\frac{i p}{m} \sum_{s=0} \frac{\Gamma\left(n-s+\frac{3}{2}\right)}{2 \Gamma\left(\frac{1}{2}\right)(n+1-s) !} \int_{-1}^{1} d x x^{2} \gamma^{2 s-3} \\
& =\frac{i p}{m}\left\{\sum_{s=0}^{n}\left(\frac{p^{0}}{m}\right)^{2 s-1 / 2} \frac{1}{2} \sum_{r=s+1}^{n+1} \frac{\Gamma\left(n-r+\frac{3}{2}\right) s ! \Gamma\left(r-\frac{1}{2}\right)}{\Gamma\left(\frac{1}{2}\right)(n+1-r) ! r ! \Gamma\left(s+\frac{1}{2}\right)}\right. \\
& \left.\quad+\frac{1}{2} \sum_{r=0}^{n+1} \frac{\Gamma\left(n-r+\frac{3}{2}\right) \Gamma\left(r-\frac{1}{2}\right)}{\Gamma\left(\frac{1}{2}\right) \Gamma\left(-\frac{1}{2}\right)(n+1-r) ! r !}\left(\frac{m}{p}\right)^{2}\left[\frac{m}{p} \sinh ^{-1}\left(\frac{p}{m}\right)-\frac{m}{p^{0}}\right]\right\} .
\end{aligned}
$$

The coefficient of the $\sinh ^{-1}$ term vanishes by virtue of the identity

$$
\sum_{s} \frac{\Gamma(a+\alpha-s) \Gamma(\beta+s)}{(a-s) ! s !} \equiv \frac{\Gamma(\alpha) \Gamma(\beta) \Gamma(\alpha+\beta+a)}{a ! \Gamma(\alpha+\beta)} .
$$

We then define the coefficients $a_{r}(n)$ :

and

$$
a_{r}(n) \equiv \sum_{s=r+1}^{n+1} \frac{\Gamma\left(n+1-s+\frac{1}{2}\right) \Gamma\left(s-\frac{1}{2}\right) r !}{2(n+1-s) ! s ! \Gamma\left(r+\frac{1}{2}\right) \Gamma\left(\frac{1}{2}\right)}, \quad a_{0}(n)=\frac{\Gamma\left(n+\frac{3}{2}\right)}{(n+1) ! \Gamma\left(\frac{1}{2}\right)},
$$

Thus

$$
\lambda_{n}=i \frac{p}{m} \sum_{r=0}^{n}\left(\frac{p^{0}}{m}\right)^{2 r-1} a_{r}(n)
$$

$$
\lim _{n \rightarrow 0} \xi^{2 n}\left(\zeta_{+}{ }^{-n-2}-\zeta_{-}{ }^{-n-2}\right)=-i\left(\frac{\mathbf{p}}{m} \cdot \boldsymbol{\nabla}\right) \delta(\mathbf{r}) \sum_{r=0}^{n}\left(\frac{p^{0}}{m}\right)^{2 r-1} a_{r}(n)
$$

We now proceed to the first time derivatives. There are two functions which contribute: $\partial^{0} \xi^{2 n} / \zeta_{ \pm}{ }^{n+1}$ and $\partial^{0} \xi^{2 n} / \zeta_{ \pm}{ }^{n+2}$. The first contributes to $\delta(\mathbf{r})$, the second has a divergent term and a finite term of the form $\nabla \nabla \delta(\mathbf{r})$. The coefficient of $\delta(\mathbf{r})$ is obtained by integrating the first function

$$
\begin{aligned}
\lim _{n \rightarrow 0} \int d^{3} r & (2 \pi)^{-2} \partial^{0}\left[\xi^{2 n}\left(\zeta_{+}^{-n-1}-\zeta_{-}^{-n-1}\right)\right] \\
= & 4 i \int d^{3} r(2 \pi)^{-2}\left[\left(\frac{p^{0} \eta}{m}\right)(n+1) \xi^{2 n} \zeta_{+}^{-n-2}-i\left(\frac{\mathbf{p} \cdot \mathbf{r}}{m}\right) \frac{p^{0}}{m} n \xi^{2(n-1)} \zeta_{+}^{-n-1}\right] \\
= & i \pi^{-1} \int_{-1}^{1} d x \gamma^{-3} \int_{-\pi / 2}^{\pi / 2} d \theta\left\{\left(\frac{p^{0}}{m}\right)(n+1)\left[\gamma \sin \theta+i\left(\frac{p}{m}\right) x \cos \theta\right]^{2(n+1)}+2 i\left(\frac{p^{2}}{m^{2}}\right) n \sum_{t=0}^{n-1}\left(\frac{p^{0}}{m}\right)^{2 t} a_{t}(n-1)\right\} \\
& =\frac{2 i \Gamma\left(n+\frac{3}{2}\right)}{n ! \Gamma\left(\frac{1}{2}\right)}+2 i\left(\frac{p^{2}}{m^{2}}\right) n \sum_{t=0}^{n-1}\left(\frac{p^{0}}{m}\right)^{2 t} a_{t}(n-1)
\end{aligned}
$$


The divergent term of $\partial^{0}\left[\xi^{2 n}\left(\zeta_{+}{ }^{-n-2}-\zeta_{-}^{-n-2}\right)\right]$ is obtained by integrating the expression

$$
\begin{aligned}
& \int d^{3} r(2 \pi)^{-2} \partial^{0}\left[\xi^{2 n}\left(\zeta_{+}{ }^{-n-2}-\zeta_{-}^{-n-2}\right)\right] \\
& =4 i \int d^{3} r(2 \pi)^{-2}\left[(n+2) \eta^{0} \xi^{2 n} \zeta^{-n-3}-i n\left(\frac{p^{0} \mathbf{p} \cdot \mathbf{r}}{m^{2}}\right) \xi^{2(n-1)} \xi^{n-2}\right] \\
& =\frac{i p^{0}}{\eta^{2} m} \int_{-1}^{1} d x \gamma^{-5} \int_{-\pi / 2}^{\pi / 2} d \theta \pi^{-1}\left\{(n+2) \cos ^{2} \theta\left[\gamma \sin \theta+i\left(\frac{p x}{p}\right) \cos \theta\right]^{2(n+1)}-i\left(\frac{p}{m}\right) x \cos \theta n\left[\gamma \sin \theta+i\left(\frac{p^{x}}{m}\right) \cos \theta\right]^{2 n+1}\right\} \\
& =i \eta^{-2}\left(\frac{p^{0}}{m}\right) \frac{\Gamma\left(n+\frac{3}{2}\right)}{(n+1) ! \Gamma\left(\frac{1}{2}\right)} \int_{-1}^{1} d x\left(\frac{3}{2} \gamma^{-5}-\gamma^{-3}\right)=i \eta^{-2}\left(\frac{m}{p^{0}}\right)^{2} \frac{\Gamma\left(n+\frac{3}{2}\right)}{(n+1) ! \Gamma\left(\frac{1}{2}\right)} .
\end{aligned}
$$

It is equally straightforward to calculate the second derivative terms, $\nabla \nabla \delta$, using the same integral with $r^{2}$ and $\xi^{2}$; we leave this as an exercise for the reader. Taking the total $\eta^{-2}$ contribution, we sum over $n$ using Eq. (4.11) to find

$$
\left(\frac{1}{\eta^{2}}\right)\left(\frac{m}{p^{0}}\right)^{2} \sum_{n} \frac{\bar{g}^{(n)}(-n)}{n+1} \Gamma(n+2) \Gamma(n+2)\left(\frac{1}{4} m^{2}\right)^{-n-1} \frac{\Gamma\left(n+\frac{3}{2}\right)}{(n+1) ! \Gamma\left(\frac{1}{2}\right)} .
$$

The condition for this to vanish is

$$
\sum_{n} \bar{g}^{(n)}(-n)\left(\frac{4}{m^{2}}\right)^{n+1} \frac{\Gamma\left(n+\frac{3}{2}\right) n !}{\Gamma\left(\frac{1}{2}\right)}=2 \Sigma \bar{g}^{(n)}(-n) m^{-2(n+1)}(2 n+1) !=0 .
$$

This same condition also removes the $1 / p^{0}$ term in Eq. (B4) and similar terms in the higher-order derivative expressions; hence it also guarantees that the commutators transform as tensors.

The second time derivatives are required for the space-space components of the commutators. Thus, we need

$$
\begin{gathered}
(2 \pi)^{-2} \lim _{n \rightarrow 0}\left(\partial^{0}\right)^{2}\left[\xi^{2 n}\left(\zeta_{+}{ }^{-n-1}-\zeta_{-}{ }^{-n-1}\right)\right]=(2 \pi)^{-2} \lim _{n \rightarrow 0}\left(-\partial^{2}\right)\left[\xi^{2 n}\left(\zeta_{+}{ }^{-n-1}-\zeta_{-}^{-n-1}\right)\right]=-\hat{p} \cdot \mathbf{r} \delta(\mathbf{r}) \lambda_{n}, \\
\bar{\lambda}_{n}=(2 \pi)^{-2} \int d^{3} r \hat{p} \cdot \mathbf{r}\left(-\partial^{2}\right)\left[\xi^{2 n}\left(\zeta_{+}{ }^{-n-1}-\zeta_{-}{ }^{-n-1}\right)\right]=-2(2 \pi)^{-2} \int d^{3} r \hat{p} \cdot \mathbf{r} \\
\times\left(2 n(2 n+1) \frac{\xi^{2(n-1)}}{\zeta^{n+1}}-4 n(n+1) \frac{\xi^{2 n}}{\zeta^{n+2}}\right)=\left[-2 n(2 n+1) \lambda_{n-1}+4 n(n+1) \lambda_{n}\right](i p / m) \\
=(i p / m) 4 n \sum_{r=0}\left(p^{0} / m\right)^{2 r-1}\left[(n+1) a_{r}(n)-\left(n+\frac{1}{2}\right) a_{r}(n-1)\right] \equiv(i p / m) 4 n \sum_{r=0}\left(p^{0} / m\right)^{2 r-1} \bar{a}_{r}(n),
\end{gathered}
$$

and

$$
\lim _{n \rightarrow 0}(2 \pi)^{-2}\left(-\partial^{2}\right)\left[\xi^{2 n}\left(\zeta_{+}^{-(n+1)}-\zeta_{-}^{-(n+1)}\right)\right]=-i\left(\frac{\mathbf{p} \cdot \nabla}{m}\right) \delta(\mathbf{r}) \sum_{r=1}\left(\frac{p^{0}}{m}\right)^{2 r-1} \bar{a}_{r}(n) .
$$

The $\left(\partial^{0}\right)^{2} \xi^{2 n} \xi^{n-2}$ term contributes third derivatives of a $\delta$ function as well as singular terms. We shall not calculate the third derivative terms, only the divergen $i$ term which is given by

$$
\begin{gathered}
\int d^{3} r(2 \pi)^{-2}(\hat{p} \cdot \mathbf{r})\left(-\partial^{2}\right)\left[\xi^{2 n}\left(\zeta_{+}{ }^{-n-2}-\zeta_{-}{ }^{-n-2}\right)\right]=2 \int d^{3} r(2 \pi)^{-2} \hat{p} \cdot \mathbf{r}\left[4(n-1)(n+2) \xi^{2 n} \zeta^{-n-3}-2 n(2 n+1) \xi^{2(n-1)} \xi^{-n-2}\right] \\
=\frac{1}{2} \eta^{-2} \int_{-1}^{1} d x x \gamma^{-5} \int_{-\pi / 2}^{\pi / 2} d \theta \pi^{-1}\left\{4(n-1)(n+2) \cos \theta[\gamma \sin \theta+i(p x / m) \cos \theta]^{2 n+3}-2 n(2 n+1) \cos \theta\right. \\
\left.\times[\gamma \sin \theta+i(p x / m) \cos \theta]^{2 n+1}\right\} \\
=i(p / m) \eta^{-2} \int_{-1}^{1} d x x^{2} \gamma^{-5}\left(\frac{2(n-1) \Gamma\left(n+\frac{5}{2}\right)}{(n+1) ! \Gamma\left(\frac{1}{2}\right)}-\frac{n(2 n+1) \Gamma\left(n+\frac{3}{2}\right)}{(n+1) ! \Gamma\left(\frac{1}{2}\right)}\right)=-2 i(p / m) \eta^{-2} \frac{\Gamma\left(n+\frac{3}{2}\right)}{(n+1) ! \Gamma\left(\frac{1}{2}\right)}\left(\frac{m}{p^{0}}\right)^{3}
\end{gathered}
$$

The coefficient of $1 / \eta^{2}$ is thus identical to that in the first derivative term, Eq. (B1). The vanishing of these terms then leads to the constraint on the $\bar{g}^{(n)}(-n)$. 
The remaining quantities of interest are the third time derivatives which have contributions to the $\delta$ function from $\ln \zeta$ and $\xi^{2 n} \zeta^{-n}$ and singular contributions from $\xi^{2 n} \zeta^{-n-1}$. The $\delta$-function contribution from $\ln \zeta$ is obtained from

where the coefficient $C$ is the integral

$$
\partial^{0}\left(-\partial^{2}\right) \ln \zeta=4(2 \pi)^{2} C \delta(\mathbf{r}),
$$

$$
C=\int d^{3} r(4 \pi)^{-2} \partial^{0}\left(-\partial^{2}\right) \ln \left(\zeta_{+} / \zeta_{-}\right)=\int d^{3} r(2 \pi)^{-2} \partial^{0}\left(1 / \zeta_{+}\right)=i ;
$$

the $\delta$-function coefficient from $\xi^{2 n} \xi^{-n}$ is similarly given by

$$
\begin{aligned}
& \int d^{3} r(2 \pi)^{-2} \partial^{0}\left(-\partial^{2}\right)\left[\xi^{2 n}\left(\zeta_{+}^{n}-\zeta_{-}{ }^{n}\right)\right]=4 n \int d^{3} r(2 \pi)^{-2} \partial^{0}\left\{2(n+1) \xi^{2 n} \zeta^{n-1}-(2 n+1) \xi^{(2 n-1)} \zeta^{-n}\right\} \\
& =4 i n\left\{2(n+1)\left[\frac{\Gamma\left(n+\frac{3}{2}\right)}{n ! \Gamma\left(\frac{1}{2}\right)}+n\left(\frac{p^{2}}{m^{2}}\right) \sum_{t=0}^{n-1}\left(\frac{p^{0}}{m}\right)^{2 t} a_{t}(n-1)\right]\right. \\
& \left.\quad-(2 n+1)\left[\frac{\Gamma\left(n+\frac{1}{2}\right)}{(n-1) ! \Gamma\left(\frac{1}{2}\right)}+(n-1)\left(\frac{p^{2}}{m^{2}}\right) \sum_{t=0}^{n-2}\left(\frac{p^{0}}{m}\right)^{2 t} a_{t}(n-2)\right]\right\} \\
& =i\left[\frac{8 \Gamma\left(n+\frac{3}{2}\right)}{(n-1) ! \Gamma\left(\frac{1}{2}\right)}+4\left(\frac{p^{2}}{m^{2}}\right) n \sum_{t=0}^{n-1}\left(\frac{p^{0}}{m}\right)^{2 t}\left[2 n(n+1) a_{t}(n-1)-(n-1)(2 n+1) a_{t}(n-2)\right]\right] .
\end{aligned}
$$

The singular terms from $\xi^{2 n} \zeta^{-n-1}$ are

from Eq. (B6).

$$
\int d^{3} r \partial^{0}\left(-\partial^{2}\right)\left[\xi^{2 n}\left(\zeta_{+}{ }^{-n-1}-\zeta_{-}^{-n-1}\right)\right] \equiv 0
$$

PHYSICAL REVIEW

VOLUME 175 , NUMBER 5

25 NOVEMBER 1968

\title{
Puzzle of Regge Dips
}

\author{
RALPH ROSKIES* $\dagger$ \\ Department of Nuclear Physics, Weizmann Institute of Science, Rehovoth, Israel
}

(Received 8 July 1968)

\begin{abstract}
The formalism giving rise to Regge dips is reviewed, and the modifications due to singular residues are discussed. The $\pi N$ charge-exchange reaction is parametrized to allow for a singular $\rho$ residue as $\alpha_{\rho}$ passes through zero, but the fits show that the singularity is very weak. A model of a singular residue is presented, and it is argued that perturbation-theory models are at variance with the experimentally observed behavior of the $\rho$ residue.
\end{abstract}

\section{INTRODUCTION}

$\mathrm{O}$ $\mathrm{NE}$ of the striking successes of Regge-pole theory has been the prediction of a dip ${ }^{1}$ in the differential cross section of $\pi N$ charge exchange (CEX) at a value of $t$ for which the $\rho$ trajectory passes through zero. Implicit in this analysis is the assumption that the residue of the $\rho$ trajectory cannot become singular at this point. However, Jones and Teplitz $z^{2}$ and Mandelstam and $W_{a n g}{ }^{3}$ have shown that such singular residues

\footnotetext{
* Canadian Society for the Weizmann Institute Fellow. $\dagger$ Present address: Yale University, New Haven, Conn.

1 F. Arbab and C. B. Chiu, Phys. Rev. 147, 1045 (1966).

${ }^{2}$ C. E. Jones and V. L. Teplitz, Phys. Rev. 159, 1271 (1967), hereafter referred to as JT.

${ }^{3}$ S. Mandelstam and L. L. Wang, Phys. Rev. 160, 1490 (1967),
} hereafter referred to as MW. are quite consistent with the concepts of unitarity and analyticity. It would seem worthwhile, then, to restudy the question of dips in this new light.

Both JT and MW have shown that in the presence of a third double-spectral function (tdsf), fixed poles are to be expected at nonsense values of the wrong signature. The conflict with elastic unitarity is removed by a moving cut which covers up the elastic region as $J$ approaches the first nonsense value of the wrong signature. Moreover, the cut also overcomes the difficulties of the Gribov-Pomeranchuk ${ }^{4}$ phenomenon.

The presence of the fixed pole depends on the existence of the tdsf. Arguing that the tdsf might be ignored,

${ }^{4} \mathrm{~V}$. Gribov and I. Ya. Pomeranchuk, Phys. Letters 2, 239 (1967). 